\title{
Numerical solution of the conditionally averaged equations for representing net mass flux due to convection
}

Article

Accepted Version

Weller, H. and Mclntyre, W. A. (2019) Numerical solution of the conditionally averaged equations for representing net mass flux due to convection. Quarterly Journal of the Royal Meteorological Society, 145 (721). pp. 1337-1353. ISSN 1477870X doi: https://doi.org/10.1002/qj.3490 Available at https://centaur.reading.ac.uk/81686/

It is advisable to refer to the publisher's version if you intend to cite from the work. See Guidance on citing.

To link to this article DOI: http://dx.doi.org/10.1002/qj.3490

Publisher: Royal Meteorological Society

All outputs in CentAUR are protected by Intellectual Property Rights law, including copyright law. Copyright and IPR is retained by the creators or other copyright holders. Terms and conditions for use of this material are defined in the End User Agreement.

www.reading.ac.uk/centaur 
Central Archive at the University of Reading

Reading's research outputs online 


\title{
Numerical Solution of the Conditionally Averaged Equations for Representing Net Mass Flux due to Convection
}

\author{
Hilary Weller, ${ }^{\mathrm{a} *}$ William McIntyre ${ }^{\mathrm{a}}$ \\ ${ }^{a}$ Meteorology, University of Reading \\ *Correspondence to: E-mail: <h.weller@ reading.ac.uk>
}

\begin{abstract}
The representation of sub-grid scale convection is a weak aspect of weather and climate prediction models and the assumption that no net mass is transported by convection in parameterisations is increasingly unrealistic as models enter the grey zone, partially resolving convection. The solution of conditionally averaged equations of motion (multi-fluid equations) is proposed in order to avoid this assumption. Separate continuity, temperature and momentum equations are solved for inside and outside convective plumes which interact via mass transfer terms, drag and by a common pressure. This is not a convection scheme that can be used with an existing dynamical core - this requires a whole new model.

This paper presents stable numerical methods for solving the multi-fluid equations including large transfer terms between the environment and plume fluids. Without transfer terms the two fluids are not sufficiently coupled and solutions diverge. Two transfer terms are presented which couple the fluids together in order to stabilise the model: diffusion of mass between the fluids (similar to turbulent entrainment) and drag between the fluids. Transfer terms are also proposed to move buoyant air into the plume fluid and vice-versa as would be needed to represent initialisation and termination of sub-grid-scale convection. The transfer terms are limited (clipped in size) and solved implicitly in order to achieve bounded, stable solutions. Results are presented of a well resolved warm bubble with rising air being transferred to the plume fluid. For stability, equations are formulated in advective rather than flux form and solved using bounded finite volume methods. Discretisation choices are made to preserve boundedness and conservation of momentum and energy when mass is transferred between fluids.
\end{abstract}

The formulation of transfer terms in order to represent sub-grid convection is the subject of future work.

Received...

\section{Introduction}

The representation of sub-grid scale convection is arguably the weakest aspect of weather and climate prediction models (eg. Stephens et al. 2010; Sherwood et al. 2013; Holloway et al. 2014) and leads to poor predictions of weather and climate in the extratropics (eg. Lean et al. 2008) and the tropics (Chapter 8 , Solomon et al. 2007). The problem gets worse when modelling convection in the grey zone, where convection is partially resolved and so the assumptions made by most convection schemes are particularly bad (eg. Gerard and Geleyn 2005). Two specific assumptions are identified which we aim to avoid:

1. Net mass flux by convection; traditional mass flux (and other) convection schemes assume that convection does not create a net transport of mass in the vertical (eg. Gregory and Rowntree 1990). Instead mass is mixed within each column.
2. Non-equilibrium dynamics; traditional convection schemes ignore effects due to changes in time of the properties of convection (eg. Kain and Fritsch 1990).

If a convection scheme transports mass as well as heat, moisture and momentum, all of the same equations of motion should be solved both inside and outside the convective plumes. Therefore it no longer makes sense to have a convection parameterisation as an external module to a dynamical core - they should both be solving the same equations and so the same model should be used for both. Dynamical cores all have some form of implicit time stepping to control divergence, either treating acoustic waves implicitly, at least in the vertical direction (eg. Weller et al. 2013), so that they do not lead to severe time-step restrictions, or some form of implicit technique to ensure (psuedo)-incompressibility (eg. Simmons et al. 1989). Once convection schemes can transport mass, they will trigger acoustic and gravity waves and so terms of the equations related to convective mass transport must appear in whatever method that the dynamical core uses to control divergence. If a significant fraction of a grid box contains a 
convective plume, a model will become unstable if the mass transported by that plume is treated explicitly and deposited at the top of the plume in one time-step. This mass transport must be treated consistently with the mass transport of the resolved flow.

The conditional averaging (or filtering) process for convection was described by Thuburn et al. (2018) and involves multiplying each equation of motion by an indicator function and averaging over a volume (or applying a different filter). This leads to equations which are similar to those of a mass flux convection scheme but without the approximation of zero net mass flux in each column. The conditionally averaged equations also have transfer terms to transfer mass, momentum, heat and moisture between the fluids. These terms have a similar role to the closures for cloud base mass flux and convective entrainment and detrainment.

There are schemes which account for aspects of nonequilibrium dynamics (eg. Gerard and Geleyn 2005; Yano and Plant 2012; Park 2014) but fewer that allow net mass flux due to convection, (exceptions being Kuell and Bott 2008; Arakawa and $\mathrm{Wu}$ 2013). Kuell et al. (2007) and Kuell and Bott (2008) extend a mass flux convection scheme to transport mass in the vertical by creating a source term of the continuity equation due to sub-grid scale convection. Their approach is not as general or consistent as that proposed by Thuburn et al. (2018) and it is also not clear if their numerical technique will be stable for moderate time-steps. Other attempts to allow net mass flux by convection (eg. Gerard and Geleyn 2005; Arakawa and Wu 2013) have relied on statistical approximations to define the area fraction associated with convection rather than on prognostic equations, as laid out by Thuburn et al. (2018). A significant advance is the extended EDMF scheme (Tan et al. 2018) which presents conditionally averaged equations of motion with different fluids for the environment and convective plume, including transport equations for the plume area fraction. Tan et al. (2018) combine conditional averaging and Reynolds averaging, presenting transport equations for sub-grid scale variability in each fluid. However the numerical solutions that they present are in a single column and they assume that no net mass is transferred out of the column in order to simplify their numerical solution. In order to make full use of the extended EDMF scheme, a robust numerical method is needed to solve conditionally averaged equations for convection in three dimensions.

Conditional averaging has been used in other fields for decades; Dopazo (1977) described how it could be used for representing intermittent turbulent flows but it has more commonly been used to represent multiphase flow (eg. Lance and Bataille 1991; Guelfi et al. 2007) with separate fluids for different phases which share a single pressure. The conditionally averaged Euler equations with a single pressure and without transfers between the fluids are in fact ill-posed (Stewart and Wendroff 1984) and are usually regularised by including coupling between phases such as drag and other relaxation transfers. Alternatively, Holm and Kupershmidt (1984) regularised these equations using multiple pressures for problems with surface tension. Thuburn et al. (2019) are also working on a single column solution of conditionally averaged equations and show that the incompressible conditionally averaged equations are unstable without additional coupling between the fluids.

This paper presents a stable numerical method for solving the conditionally averaged equations in arbitrary dimensions and proposes transfer terms that transfer resolved convection into the buoyant fluid and stable air back into the stable fluid. These transfer terms are not designed to be used to represent subgrid scale convection as this would require more information about sub-grid scale variability. Instead they are designed to be large source terms that will act to challenge the stability of the numerical method as they are too big to be treated explicitly and they will introduce new mass, temperature and momentum extrema in one fluid. We use two techniques to regularise the multifluid equations; the first is with drag between fluids and the second is with diffusion between the fluids, similar to lateral entrainment and detrainment.

\section{The Conditionally Averaged Euler Equations}

Traditional mass flux convection schemes solve simplified equations of motion with temperature, vertical velocity and moisture inside convective plumes. This is therefore a form of conditional averaging with variables averaged inside and outside plumes. However we can take the process further and avoid some of the crude assumptions made by mass flux schemes such as vanishing convective area fraction and no net mass flux due to convection. The conditional averaging (or filtering) process for convection was described by Thuburn et al. (2018) and involves multiplying each equation of motion by an indicator function, $I_{i}$, for a number of different conditions labelled by $i$. At each point, $I_{i}$ is one if conditions $i$ is true and zero otherwise. Fluid $i=0$ may be for example the stable environment, fluid $i=1$ convective plumes and fluid $i=2$ may be downdrafts. A filter (typically volume average) is then applied and averages for each condition can be found over each filter region. The volume fraction in fluid $i$ is defined to be

$$
\sigma_{i}=\widetilde{I}_{i}
$$

where the ${ }^{\sim}$ implies the application of the filter (or volume average). Density, potential temperature and velocity can then be defined for each fluid:

$$
\begin{aligned}
\rho_{i} & =\widetilde{I_{i} \rho} / \sigma_{i} \\
\theta_{i} & =\widetilde{I_{i} \rho \theta} /\left(\sigma_{i} \rho_{i}\right) \\
\mathbf{u}_{i} & =\widetilde{I_{i} \rho \mathbf{u}} /\left(\sigma_{i} \rho_{i}\right)
\end{aligned}
$$

and averages over all fluids (denoted by overbar) are:

$$
\begin{aligned}
1 & =\sum_{i} \sigma_{i} \\
\bar{\rho} & =\sum_{i} \sigma_{i} \rho_{i} \\
\overline{\rho \theta} & =\sum_{i} \sigma_{i} \rho_{i} \theta_{i} \\
\overline{\rho \mathbf{u}_{i}} & =\sum_{i} \sigma_{i} \rho_{i} \mathbf{u}_{i} .
\end{aligned}
$$

As with Reynolds or Favre averaging, non-linear conditionally averaged terms such as $\widehat{I_{i} \rho \mathbf{u} \theta}$ and $\widehat{I_{i} \rho \mathbf{u u}}$ are not equal to the products of conditionally averaged terms. The difference is expressed as a sub-filter scale flux:

$$
\begin{aligned}
\widetilde{I_{i} \rho \mathbf{u} \theta} & =\sigma_{i} \rho_{i} \mathbf{u}_{i} \theta_{i}+\mathbf{F}_{\mathrm{SF}}^{\theta_{i}} \\
\widetilde{I_{i} \rho \mathbf{u u}} & =\sigma_{i} \rho_{i} \mathbf{u}_{i} \mathbf{u}_{i}+\mathrm{F}_{\mathrm{SF}}^{\mathbf{u}_{i}}
\end{aligned}
$$

where $\mathbf{F}_{\mathrm{SF}}^{\theta_{i}}$ is a vector and $\mathrm{F}_{\mathrm{SF}}^{\mathbf{u}_{i}}$ is a tensor. The sub-filter scale fluxes could be due to turbulent motions within each fluid. Parameterisations for sub-filter scale fluxes are not considered in this paper. We are still able to find stable solutions of the equations because the flow that we are solving is well resolved.

The same averaging can be applied to pressure but we will assume that pressure is uniform across all fluids. Pressure differences between the plume and the environment will be parameterised as form drag which is discussed in section 2.1.1. 
Conditional averaging is also used to represent multi-phase flows (eg. Guelfi et al. 2007) and uniform pressure across phases is assumed. A single pressure for compressible multiphase flows is known to lead to an ill-posed problem (Stewart and Wendroff 1984) but the equations can be regularised with some kind of coupling between fluids which will be discussed in section 2.1. Tan et al. (2018) do not assume that the pressure is equal in each fluid but they do assume that density is equal in both fluids, except where it influences buoyancy. Tan et al. (2018) also assume that drag is high enough that horizontal velocities are equal between fluids.

Conditional averaging is applied to the dry, adiabatic, rotating, compressible Euler equations in flux form, assuming uniform pressure between fluids and ignoring sub-fiter scale fluxes. This leads to the following conditionally averaged Euler equations for mass, potential temperature and momentum:

$$
\begin{aligned}
\frac{\partial \sigma_{i} \rho_{i}}{\partial t} & +\nabla \cdot\left(\sigma_{i} \rho_{i} \mathbf{u}_{i}\right)=\sum_{j \neq i}\left(\sigma_{j} \rho_{j} S_{j i}-\sigma_{i} \rho_{i} S_{i j}\right) \\
\frac{\partial \sigma_{i} \rho_{i} \theta_{i}}{\partial t} & +\nabla \cdot\left(\sigma_{i} \rho_{i} \mathbf{u}_{i} \theta_{i}\right) \\
& =\sum_{j \neq i}\left(\sigma_{j} \rho_{j} \theta_{j} S_{j i}-\sigma_{i} \rho_{i} \theta_{i} S_{i j}-\sigma_{i} \rho_{i} H_{i j}\right) \\
\frac{\partial \sigma_{i} \rho_{i} \mathbf{u}_{i}}{\partial t} & +\nabla \cdot\left(\sigma_{i} \rho_{i} \mathbf{u}_{i} \mathbf{u}_{i}\right)=-2 \sigma_{i} \rho_{i} \boldsymbol{\Omega} \times \mathbf{u}_{i} \\
& -\sigma_{i} \rho_{i} c_{p} \theta_{i} \nabla \pi+\sigma_{i} \rho_{i} \mathbf{g} \\
& +\sum_{j \neq i}\left(\sigma_{j} \rho_{j} \mathbf{u}_{j} S_{j i}-\sigma_{i} \rho_{i} \mathbf{u}_{i} S_{i j}-\sigma_{i} \sigma_{j} \mathbf{d}_{i j}\right)
\end{aligned}
$$

where $\pi=\left(p / p_{0}\right)^{\kappa}$ is the Exner pressure, $p$ is the pressure, $p_{0}$ is a reference pressure, $\kappa=R / c_{p}, R$ is the gas constant of dry air, $c_{p}$ is the heat capacity of dry air at constant pressure, $\theta=T / \pi$ is the potential temperature, $\Omega$ is the rotation rate of the domain, $\mathbf{g}$ is the acceleration due to gravity, $\sigma_{i} \rho_{i} S_{i j}$ is the transfer rate of mass from fluid $i$ to fluid $j, \mathbf{d}_{i j}$ is the drag exerted from fluid $i$ onto fluid $j$ and $H_{i j}$ is the heat transfer from fluid $i$ to $j$. Formulation of the transfer terms, $S_{i j}, H_{i j}$ and $\mathbf{d}_{i j}$ is needed to create a parameterisation of convection. The mass transfer, $S_{i j}$, will be dependent on the definitions of each fluid and on sub-grid scale variability. Heat transfer and drag between fluids, $H_{i j}$ and $\mathbf{d}_{i j}$, will depend on the surface area between fluids. When mass is transferred, we assume that its mean properties are taken with it which is why $S_{i j}$ appears in all equations. We then also assume the equation of state for dry air both globally and for each fluid is given by:

$$
p_{0} \pi^{\frac{1-\kappa}{\kappa}}=R \rho_{i} \theta_{i}=R \overline{\rho \theta}=R \sum_{i} \sigma_{i} \rho_{i} \theta_{i} .
$$

The temperature and momentum equations can be expressed in advective form so that the primitive variables $\mathbf{u}_{i}$ and $\theta_{i}$ are well defined when $\sigma_{i}$ is zero:

$$
\begin{aligned}
\frac{\partial \theta_{i}}{\partial t} & +\mathbf{u}_{i} \cdot \nabla \theta_{i}=\sum_{j \neq i}\left(\frac{\sigma_{j} \rho_{j}}{\sigma_{i} \rho_{i}} S_{j i}\left(\theta_{j}-\theta_{i}\right)-H_{i j}\right) \\
\frac{\partial \mathbf{u}_{i}}{\partial t} & +\mathbf{u}_{i} \cdot \nabla \mathbf{u}_{i}=-2 \boldsymbol{\Omega} \times \mathbf{u}_{i}-c_{p} \theta_{i} \nabla \pi+\mathbf{g} \\
& +\sum_{j \neq i}\left(\frac{\sigma_{j} \rho_{j}}{\sigma_{i} \rho_{i}} S_{j i}\left(\mathbf{u}_{j}-\mathbf{u}_{i}\right)-\mathbf{D}_{i j}\right)
\end{aligned}
$$

where $\mathbf{D}_{i j}=\sigma_{j} \mathbf{d}_{i j} / \rho_{i}$. Note that if $\sigma_{i}$ is zero, there is a division by zero in the mass transfer terms in eqns (15) and (16) which leads to an infinite source term when $\theta$ and $\mathbf{u}$ are transferred to an empty fluid. This is appropriate because when mass is transferred to an empty fluid, the properties should instantaneously become those of the transferred fluid; the old properties of the empty fluid should not have any influence. However this infinite source term will require careful numerical treatment.

\subsection{Transfers and Exchanges between Fluids}

In order to represent sub-grid scale convection with the multi-fluid equations we first need to give labels to parts of the fluid with different properties. For example, fluid zero could be the stable environment fluid, fluid one could be the convectively unstable buoyant plumes and fluid two could be downdrafts. For now it doesn't matter precisely how this is done. The next essential stage is to formulate transfer terms, $\mathbf{D}_{i j}, S_{i j}$ and $H_{i j} . S_{i j}$ is particularly important for moving mass in and out of the fluid related to convection and will presumably be associated with sub-grid scale variability of buoyancy and other properties related to convection. In this paper we do not propose new closures for how mass moves from the stable to the convectively active fluid (such as cloud base mass flux). Instead we will use transfer terms formulated in terms of differential operators that transfer fluid based on the properties of the resolved flow, neglecting sub-grid-scale variability. The purpose of this is to test the stability, boundedness and conservation properties of the numerical methods rather than proposing a useful parameterisation of convection.

We also include transfer terms to stabilise the equations. For further justification of the need for stabilisation, we should consider the physical meaning of a mixture of fluids. $0<\sigma_{0}<1$ implies that at least two fluids are present at scales down to the grid-scale. This implies that there will be a large surface area between the two fluids and so they are likely to exchange mass and momentum. We therefore couple the two fluids using mass exchanges or drag between the fluids. Sensible and radiative heat transfer between fluids is not considered in this paper and appears not to be needed for stability because the temperature of the fluids does not diverge.

We will consider one form of drag and three types of mass transfer. The total mass transfer from fluid $i$ to $j$ is:

$$
S_{i j}=S_{d i j}+S_{b i j}+S_{h i j}
$$

where $S_{d i j}$ is due to diffusion of $\sigma, S_{b i j}$ is due to buoyancy perturbations and $S_{h i j}$ is due to horizontal divergence. $S_{b i j}$ and $S_{h i j}$ are forms of cloud base mass flux. The diffusion of $\sigma$, $S_{d i j}$, mixes the fluids in regions where the gradient of $\sigma$ varies rapidly. $S_{d i j}$ could be similar to lateral entrainment and is used to regularise the ill-posed equations. These and the drag term will be described next. Throughout we assume $H_{i j}=0$.

\subsubsection{Drag in the Momentum Equation}

Pressure differences between the fluid are parameterized as form drag. We use a model for the drag on a rising bubble based on Roghair et al. (2011) which is similar to the form used by Simpson and Wiggert (1969) for modelling buoyant plumes and the form recommended by Romps and Charn (2015) for deep convection. Assuming exactly two fluids and remembering that we need $\sigma_{i} \rho_{i} \mathbf{D}_{i j}=-\sigma_{j} \rho_{j} \mathbf{D}_{j i}$ we can use:

$$
\mathbf{D}_{i j}=\frac{\sigma_{j}}{\rho_{i}} \frac{C_{D} \rho_{i j}}{r_{c}}\left|\mathbf{u}_{i}-\mathbf{u}_{j}\right|\left(\mathbf{u}_{i}-\mathbf{u}_{j}\right)
$$

where $C_{D}$ is a drag coefficient, $r_{c}$ is a radius and $\rho_{i j}=\left(\sigma_{i} \rho_{i}+\right.$ $\left.\sigma_{j} \rho_{j}\right) /\left(\sigma_{i}+\sigma_{j}\right)$. The radius, $r_{c}$, needs to be the same for both fluids and could be, for example, the plume radius or a length scale for the region of fluid with lower volume fraction. Initial testing without any form of stabilisation showed that the velocity of the vanishing fluid diverges which is clearly unrealistic - a tiny bubble of a different fluid would move with the main fluid. This can be achieved by making $r_{c}$ small as $\sigma_{i}$ becomes small in any fluid which increases the drag between fluids. If we assume a maximum 
and minimum radius, $r_{\max }$ and $r_{\min }$, then $r_{c}$ can take the form:

$$
r_{c}=\max \left(r_{\min }, \sigma_{i} \sigma_{j} r_{\max }\right)
$$

For a more realistic scheme, future work could draw on existing parameterisations of cloud radius. From geometric arguments, it is reasonable to expect $r_{c}$ to be small when $\sigma$ is small but stability restrictions on $r_{c}$ are not known.

\subsubsection{Diffusion of $\sigma$}

A diffusive mass transfer term is created to smooth out high curvature or oscillations in $\sigma$. The form used ensures that total mass is not diffused and that mass transfer terms are never negative:

$$
\sigma_{i} \rho_{i} S_{d i j}=\frac{K_{\sigma}}{2} \max \left(\nabla^{2}\left(\sigma_{j} \rho_{j}-\sigma_{i} \rho_{i}\right), 0\right)
$$

where $K_{\sigma}$ is a diffusion coefficient which can be chosen so as to obey stability constraints if the mass transfer term is treated explicitly in equation (11). This mixing of the fluids is similar to turbulent entrainment at the lateral edges of plumes but eqn (20) is different from entrainment rates in other schemes which depend on buoyancy and vertical velocity (eg. Tan et al. 2018) or on plume radius (eg. de Rooy et al. 2013). In future work, existing parameterisations for entrainment could be used to set $K_{\sigma}$.

\subsubsection{Transfers based on buoyancy perturbations}

This transfer term has only been derived considering two fluids: the stable environment $(i=0)$ and the buoyant plume $(i=1)$. It is formulated to mimic a parameterisation for cloud base mass flux rather than to regularise the equations: transfer from fluid $i=0$ to fluid $i=1$ could introduce new extrema of $\sigma_{i}, \theta_{1}$ and $\mathbf{u}_{1}$. Air will rise if buoyancy perturbations make it lighter than the air above or lighter that the surroundings. These observations are usually used in closures for cloud base mass flux by testing the (conditional) stability of a parcel (eg. Gregory and Rowntree 1990). We want to formulate transfer terms in terms of PDEs rather than introducing criteria comparing the buoyancy of grid boxes with surrounding grid boxes. Therefore we use the Laplacian of $\theta$ to inform us of positive and negative perturbations. There will be a positive perturbation if $\nabla^{2} \theta<0$ and vice versa. :

$$
\begin{aligned}
S_{b 01} & = \begin{cases}-K_{\theta} \frac{\nabla^{2} \theta_{0}}{\theta_{0}} & \text { when } \nabla^{2} \theta_{0}<0 \\
0 & \text { otherwise }\end{cases} \\
S_{b 10} & = \begin{cases}K_{\theta} \frac{\nabla^{2} \theta_{1}}{\theta_{1}} & \text { when } \nabla^{2} \theta_{1}>0 \\
0 & \text { otherwise }\end{cases}
\end{aligned}
$$

where $K_{\theta}$ is a diffusivity. These transfer terms do not guarantee that fluid 0 is stable or that fluid 1 is buoyant but that is the ultimate aim.

\subsubsection{Transfers Based on Horizontal Divergence and Vertical Velocity}

This transfer term has also been derived considering only two fluids, the stable environment $(i=0)$ and the buoyant plume $(i=1)$. It is also formulated to mimic convection rather than to regularise the equations. The transfer term moves fluid from fluid zero to one when fluid zero is converging in the horizontal and rising, and moves fluid from fluid one to fluid zero when fluid one is diverging in the horizontal and falling:

$$
\begin{aligned}
& \sigma_{0} \rho_{0} S_{h 01}= \begin{cases}-\nabla_{h} \cdot\left(\sigma_{0} \rho_{0} \mathbf{u}_{0}\right) & \text { if } \nabla_{h} \cdot\left(\sigma_{0} \rho_{0} \mathbf{u}_{0}\right)<0 \\
\text { and } \mathbf{u}_{0} \cdot \mathbf{g}<0 & \text { otherwise }\end{cases} \\
& \sigma_{1} \rho_{1} S_{h 10}= \begin{cases}\nabla_{h} \cdot\left(\sigma_{1} \rho_{1} \mathbf{u}_{1}\right) & \text { if } \nabla_{h} \cdot\left(\sigma_{1} \rho_{1} \mathbf{u}_{1}\right)>0 \\
& \text { and } \mathbf{u}_{1} \cdot \mathbf{g}>0 \\
0 & \text { otherwise }\end{cases}
\end{aligned}
$$

\section{Semi-Implicit Numerical Solution}

Solving the conditionally averaged equations needs an entire atmospheric model rather than just the convection parameterisation. Once the sub-grid-scale convection interacts with the gridscale continuity equation, convection can no longer be an isolated parameterisation - the whole model needs to change, particularly if the mass changes due to convection are to be treated implicitly, which is needed to avoid time-step restrictions based on the acoustic or gravity wave speed. Therefore the entire numerical model is described here. Aspects that are not specific to the stability of solving the conditionally averaged equations are given in appendix $\mathrm{A}$.

The equations are discretised and solved using the OpenFOAM library (https: / / openfoam.org) and the full implementation is part of the AtmosFOAM repository (https: / / github. $\mathrm{com} / \mathrm{AtmosFOAM} /$ ). The spatial discretisation uses standard OpenFOAM operators.

\subsection{Spatial Discretisation}

Most of the spatial discretisation is not specific to the stable solution of the conditionally averaged equations and is described in appendix A. The spatial discretisation uses a finite-volume Cgrid for an arbitrary mesh, similar to that described by Weller and Shahrokhi (2014) with $\theta_{i}, \sigma_{i} \rho_{i}$ and $\pi$ defined as volumetric mean quantities (or at cell centres) and normal components of velocity defined on cell faces. All the meshes used are orthogonal and, as the focus of this paper is not spatial discretisation, the discretisation is only described for orthogonal meshes for simplicity.

Specific to the conditionally averaged equations, it is important to use bounded advection of $\sigma_{i}$. A total variation diminishing (TVD) advection scheme with a van-Leer limiter is described in appendix A.4. Note that it is the advection of $\sigma_{i}$ that is bounded, not the advection of $\sigma_{i} \rho_{i}$ because it is necessary to discretise $\rho_{i}$ consistently with the pressure for both stability and energy conservation. This is described in more detail in sections 3.2.2 and 3.2.6. Bounded advection of $\rho_{i}$ is not needed because density is sufficiently smooth and far from zero that it remains positive.

\subsection{Time Stepping Algorithm}

It is crucial to get the time-stepping right for the stable solution of the conditionally averaged equations. In this section we will distinguish between convenient modelling choices and choices that are specific to the stable solution of the conditionally averaged equations. The solution of the conditionally averaged equations is interwoven with a semi-implicit method so that acoustic waves are treated implicitly for any value of $\sigma_{i}$

The terms of the Euler equations involved in acoustic waves are solved using second-order Crank-Nicolson time-stepping. Other implicit schemes could be chosen and optionally gravity waves could also be treated implicitly. For the stable solution of the conditionally averaged equations it is necessary to include updates from both the plume and environment momentum and continuity equations in the implicit solution of the pressure equation. 
Non-linear terms and advection are treated explicitly using second-order Runge-Kutta time-stepping for convenience (Weller et al. 2013). An inner loop solves the pressure equation for $\pi$ and $\mathbf{u}_{i}$ twice with explicit terms updated for the second iteration. The inner iterations are indexed with $\ell=1,2$. An outer loop solves the continuity and temperature equations explicitly for $\sigma_{i} \rho_{i}$ and $\theta_{i}$. The outer iterations are indexed with $k=1,2$. This low Mach number semi-implicit method follows Weller and Shahrokhi (2014) and is similar to Wood et al. (2014) in the number of inner and outer loops.

All transfer terms are solved using operator split either explicit or implicit first-order time-stepping. The operator splitting is a straightforward way to ensure positivity. Implicit updates are necessary for stable treatment of the large source terms (the source terms of the temperature equation (15) momentum equation (16) with $\sigma_{i} \rho_{i}$ in the denominator which can tend to infinity as $\sigma_{i} \rightarrow$ $0)$.

\subsubsection{Initialisation}

The prognostic variables are $U_{i}, \sigma_{i} \rho_{i}, \theta_{i}$ where $U_{i}=\mathbf{u}_{i} \cdot \mathbf{S}_{f}$ is the volume flux across each cell face $\left(\mathbf{S}_{f}\right.$ is the vector normal to each face with magnitude of the face area). Transport equations are solved for $U_{i}, \sigma_{i} \rho_{i}, \theta_{i}$ and $\pi$. This system is overspecified because $\pi$ can be calculated for all of the $\sigma_{i} \rho_{i}$ and $\theta_{i}$ using the equation of state. To avoid over specification, only $\mathbf{u}_{i}, \sigma_{i}, \theta_{i}$ and $\pi$ are read in at initialisation and each $\rho_{i}$ is calculated from the equation of state (14). A separate code for calculating the initial conditions calculates $\pi$ that is in discrete hydrostatic balance with $\theta_{0}$ (i.e. $\theta$ for fluid zero).

In order to start each time-step, $\left(\sigma_{i} \rho_{i}\right)^{k=0}$ is set to $\left(\sigma_{i} \rho_{i}\right)^{n}$ where $n$ is the label for the old time step, $\theta_{i}^{k=0}$ is set to $\theta_{i}^{n}, \pi^{\ell=0}$ is set to $\pi^{n}$ and $U_{i}^{\ell=0}$ to $U_{i}^{n}$. After the loops over $k$ and $\ell$, the values at time $n+1$ are set equal to the final values at the end of the iterations.

\subsubsection{Solving the Continuity Equation}

The first equations to be solved in the outer loop are the continuity equations for each $\sigma_{i} \rho_{i}(11)$. When transfer terms are included (which lead to terms with $\sigma_{i} \rho_{i}$ in the denominator of the source terms of the temperature (15) and momentum (16) equations) it is essential to keep $\sigma_{i} \rho_{i}$ positive for stability. The continuity equations are solved using operator splitting, first advecting $\sigma_{i} \rho_{i}$ and then applying the transfer terms. This is the most straightforward way to maintain positivity. We have chosen a TVD advection scheme with a van-Leer limiter (section A.4) to calculate $\sigma_{i}$ inside the divergence for iterations $k=1,2$ :

$\left(\sigma_{i} \rho_{i}\right)^{\prime}=\left(\sigma_{i} \rho_{i}\right)^{n}-\Delta t \nabla \cdot\left(\left[(1-\alpha) \rho_{i}^{n} \mathbf{u}_{i}^{n}+\alpha \rho_{i}^{k-1} \mathbf{u}_{i}^{\ell-1}\right] \sigma_{i}^{n}\right)$

where $\Delta t$ is the time-step and $\alpha$ is the off-centering parameter. For all the simulations presented, $\alpha=1 / 2$ is used making the time-stepping second-order accurate. $\rho_{i}$ inside the divergence is calculated using linear interpolation. $\left(\sigma_{i} \rho_{i}\right)^{k}$ for all values of $k$ and the intermediate values $\left(\sigma_{i} \rho_{i}\right)^{\prime}$ share the same memory as $\left(\sigma_{i} \rho_{i}\right)^{n+1}$.

Only $\sigma_{i}^{n}$ appears on the right hand side of eqn (25), no newer values such as $\sigma_{i}^{k-1}$. This is because the advection scheme assumes that the upwind values are at the old time-level and it is only guaranteed bounded when using $\sigma_{i}^{n}$ on the right hand side. However updated values of $\rho_{i}$ are used on the right hand side of eqn (25) so that the solution of eqn (25) remains consistent with the Exner pressure, $\pi$.

Next the mass transfer terms are calculated using $\sigma_{i}^{\prime}, \mathbf{u}_{i}^{\ell-1}$ and $\theta_{i}^{\ell-1}$ (the most up to date values) and limited to ensure that $\sigma_{i} \rho_{i}$ remains positive:

$$
S_{i j}^{\lim }=\frac{1}{\left(\sigma_{i} \rho_{i}\right)^{\prime}} \min \left(\left(\sigma_{i} \rho_{i}\right)^{\prime} S_{i j}, \frac{\left(\sigma_{i} \rho_{i}\right)^{\prime}-\sigma_{\min } \rho_{i}^{k-1}}{\Delta t}\right)
$$

where $\sigma_{\min }=10^{-9}$ is used in the simulations presented in section 4. Then the mass transfer is used to update $\sigma_{i} \rho_{i}$ explicitly with operator splitting:

$$
\left(\sigma_{i} \rho_{i}\right)^{k}=\left(\sigma_{i} \rho_{i}\right)^{\prime}+\Delta t\left(\left(\sigma_{j} \rho_{j}\right)^{\prime} S_{j i}^{\mathrm{lim}}-\left(\sigma_{i} \rho_{i}\right)^{\prime} S_{i j}^{\mathrm{lim}}\right) .
$$

\subsubsection{Solving the $\theta_{i}$ equation}

After the continuity equation, the $\theta_{i}$ equation (15) is solved using operator splitting; first advecting $\theta_{i}$ then applying the mass transfer terms to the advected $\theta_{i}$. Because we are using a finite volume model to solve equations in advective form, the advection of $\theta_{i}$ is calculated as:

$$
\begin{aligned}
\theta_{i}^{\prime}=\theta_{i}^{n}-\Delta t & \left((1-\alpha)\left[\nabla \cdot\left(\theta_{i} \mathbf{u}_{i}\right)-\theta_{i} \nabla \cdot \mathbf{u}_{i}\right]^{n}\right. \\
& \left.+\alpha\left[\nabla \cdot\left(\theta_{i}^{k-1} \mathbf{u}_{i}^{\ell-1}\right)-\theta_{i}^{k-1} \nabla \cdot \mathbf{u}_{i}^{\ell-1}\right]\right)
\end{aligned}
$$

where the spatial discretisation is described in section A.5.

The mass transfer terms are applied implicitly because they can be very large due to the presence of $\sigma_{i} \rho_{i}$ in the denominator. The implicit addition of mass transfer terms is formulated to be specific for having two fluids although it would be straightforward to generalise. In order to derive the equations for adding the mass transfer terms to $\theta_{i}$ we will write the $\theta_{i}$ equation as:

$$
\theta_{i}^{k}=\theta_{i}^{\prime}+\Delta t \sum_{j \neq i}\left(\frac{\left(\sigma_{j} \rho_{j}\right)^{\prime}}{\left(\sigma_{i} \rho_{i}\right)^{\prime}} S_{j i}^{\lim }\left(\theta_{j}^{k}-\theta_{i}^{k}\right)\right)
$$

Note values at level $k$ are on the left and right hand side making this an implicit solution. For $i=0,1$ this can be re-arranged to give:

$$
\begin{aligned}
\theta_{0}^{k} & =\frac{\left(1+\Delta t T_{01}\right) \theta_{0}^{\prime}+\Delta t T_{10} \theta_{1}^{\prime}}{1+\Delta t T_{10}+\Delta t T_{01}} \\
\theta_{1}^{k} & =\frac{\theta_{1}^{\prime}+\Delta t T_{01} \theta_{0}^{k}}{1+\Delta t T_{01}}
\end{aligned}
$$

where $T_{i j}=\frac{\left(\sigma_{i} \rho_{i}\right)^{\prime}}{\left(\sigma_{j} \rho_{j}\right)^{\prime}} S_{i j}^{\lim }$ is calculated just before eqn (27) so as to use $\left(\sigma_{i} \rho_{i}\right)^{\prime}$ before it is over-written by $\left(\sigma_{i} \rho_{i}\right)^{k}$. For conservation of internal energy, it is necessary that the values of $\sigma_{i} \rho_{i}$ from after advection but before the mass transfer are used in the calculation of $T_{i j}$. This ensures that $\sum_{\text {cells }} \sum_{i}\left(\sigma_{i} \rho_{i} \theta_{i}\right)^{n}=$ $\sum_{\text {cells }} \sum_{i}\left(\sigma_{i} \rho_{i} \theta_{i}\right)^{n+1}$. Calculation of $\theta_{i}^{k}$ from eqns (30) and (31) also ensures boundedness of $\theta_{i}\left(\theta_{i}^{k}\right.$ will remain between $\theta_{i}^{k-1}$ and $\left.\theta_{j}^{k-1}\right)$

\subsubsection{Diagnosing $\sigma_{i}$}

After the updates of prognostic variables $\left(\sigma_{i} \rho_{i}\right)$ and $\theta_{i}$, the diagnostic variable $\sigma_{i}$ can be updated. $\sigma_{i}$ is not used in isolation from $\rho_{i}$ anywhere in the equations $(11,14-16)$. However $\sigma_{i}$ and $\rho_{i}$ may be needed independently in closure assumptions, such as the approximation of the drag (18). Firstly, each $\rho_{i}$ is calculated from the equation of state using the most up to date values:

$$
\rho_{i}^{k}=\frac{p_{0} \pi^{\frac{1-\kappa}{\kappa}}}{R \theta_{i}}=\frac{\overline{\rho \theta}}{\theta_{i}} .
$$

Then each $\sigma_{i}$ can be calculated:

$$
\sigma_{i}^{k}=\frac{\left(\sigma_{i} \rho_{i}\right)^{k}}{\rho_{i}^{k}}
$$

This calculation will guarantee $\sum_{i} \sigma_{i}=1$. 


\subsubsection{Momentum and Continuity}

Here we describe how a standard semi-implicit algorithm is adapted to solve the momentum (16) and continuity (11) equations from all fluids simultaneously. The momentum and continuity equations are combined to form a Helmholtz equation for $\pi$. This is done by expressing the volume flux, $U_{i}$, and the mean density, $\bar{\rho}$, as linear functions of $\pi$ and substituting these into the continuity equation. This is done twice per outer loop, in an inner loop indexed by $\ell$.

The normal component of the volume flux, $U_{i}$ is expressed as a linear function of $\pi$ using the momentum equation:

$$
U_{i}^{\prime}=U_{i}^{\prime \prime}-\alpha \Delta t c_{p} \theta_{f i}^{\ell} \nabla_{S} \pi^{\ell}
$$

where $c_{p} \theta_{f i}^{\ell} \nabla_{S} \pi^{n+1}$ is an approximation of $c_{p} \theta_{i} \nabla \pi \cdot \mathbf{S}_{f}$ and is calculated from equation (60) in appendix A.2.1. $U_{i}^{\prime \prime}$ is the explicitly calculated part of the volume flux:

$$
\begin{aligned}
U_{i}^{\prime \prime} & =U_{i}^{n} \\
& -(1-\alpha) \Delta t\left(\left[\mathbf{u}_{i} \cdot \nabla \mathbf{u}_{i}+2 \boldsymbol{\Omega} \times \mathbf{u}_{i}\right] \cdot \mathbf{S}_{f}+c_{p} \theta_{f i} \nabla_{S} \pi\right)^{n} \\
& -\alpha \Delta t\left(\mathbf{u}_{i} \cdot \nabla \mathbf{u}_{i}+2 \boldsymbol{\Omega} \times \mathbf{u}_{i}\right)^{\ell-1} \cdot \mathbf{S}_{f}+\Delta t \mathbf{g} \cdot \mathbf{S}_{f}
\end{aligned}
$$

Equation (34) is multiplied by the linear interpolate of $\sigma_{i} \rho_{i}$ onto faces and then the sum is taken over all fluids to get the total mass flux:

$$
F^{\ell}=\sum_{i}\left(\sigma_{i} \rho_{i}\right)_{f}^{k} U_{i}^{\prime \prime}-\alpha \Delta t c_{p} \overline{\rho \theta}_{f}^{k} \nabla_{S} \pi^{\ell} .
$$

This will be substituted into the divergence term of the continuity equation once we have described the linear representation of $\rho$ as a function of $\pi$ which follows Weller and Shahrokhi (2014).

In order to derive a Helmholtz equation for $\pi$ using the continuity equation, the density is expressed as

$$
\bar{\rho}=\Psi \pi
$$

where $\Psi$ is the compressibility from the equation of state:

$$
\Psi=\bar{\rho}^{\frac{2 \kappa-1}{\kappa-1}}\left(\frac{R \bar{\theta}}{p_{0}}\right)^{\frac{\kappa}{\kappa-1}} .
$$

Equations (36) and (37) are substituted into the continuity equation and Gauss's divergence theorem is used to calculate the divergence term:

$$
\begin{aligned}
& \frac{\Psi^{\ell-1} \pi^{\ell}-\Psi^{n} \pi^{n}}{\Delta t}+\frac{1-\alpha}{V} \sum_{f \in c} F^{n} \\
& \frac{\alpha}{V} \sum_{f \in c}\left\{\sum_{i}\left(\sigma_{i} \rho_{i}\right)_{f}^{k} U_{i}^{\prime \prime}-\alpha \Delta t c_{p}\left(\overline{\rho \theta}_{f}^{k}\right) \nabla_{S} \pi^{\ell}\right\}=0
\end{aligned}
$$

where $V$ is the cell volume. There are no transfer terms in this equation because they cancel when summed over fluids. This is a Helmholtz equation that can be solved for $\pi^{\ell}$. Back substitutions are then made to calculate each $U_{i}^{\prime}$ using equation (34).

Applying Drag and Mass Transfer to the Momentum Equation The transfer terms of equation (16) can be applied after the solution of the Helmholtz equation because they do not directly influence the pressure. They are applied implicitly, firstorder with operator splitting with a simultaneous solution for two fluids, $i$ and $j$. $U_{i}^{\prime}$ is the volume flux predicted by the back substitution after the Helmholtz equation and $U_{i}^{\ell}$ is the solution of
$U_{i}$ after implicit application of the source terms:

$$
\begin{aligned}
U_{i}^{\ell} & =U_{i}^{\prime}-\Delta t\left(T_{j i}+\sigma_{j} \frac{C_{D} \bar{\rho}^{k}}{r_{c} \rho_{i}^{k}}\left|\mathbf{u}_{i}-\mathbf{u}_{j}\right|^{\ell-1}\right) U_{i}^{\ell} \\
& +\Delta t\left(T_{j i}+\sigma_{j} \frac{C_{D} \bar{\rho}^{k}}{r_{c} \rho_{i}^{k}}\left|\mathbf{u}_{i}-\mathbf{u}_{j}\right|^{\ell-1}\right) U_{j}^{\ell}
\end{aligned}
$$

Using the same block implicit solution technique as was described in section 3.2.3, $U_{i}^{\ell}$ can be calculated using:

$$
\begin{aligned}
U_{0}^{\ell} & =\frac{\left(1+\Delta t \mathbb{T}_{01}\right) U_{0}^{\prime}+\Delta t \mathbb{T}_{10} U_{1}^{\prime}}{1+\Delta t \mathbb{T}_{10}+\Delta t \mathbb{T}_{01}} \\
U_{1}^{\ell} & =\frac{U_{1}^{\prime}+\Delta t \mathbb{T}_{01} U_{0}^{\ell}}{1+\Delta \mathbb{T}_{01}}
\end{aligned}
$$

where $\mathbb{T}_{i j}=T_{i j}+\frac{\sigma_{i}^{k}}{\rho_{j}^{k}} \frac{C_{D} \bar{\rho}^{k}}{r_{c}}\left|\mathbf{u}_{j}-\mathbf{u}_{i}\right|^{\ell-1}$. As with the numerical method for applying the mass transfer terms to the $\theta_{i}$ equations, this technique ensures that the $U_{i}$ remain bounded and the use of the values of $\sigma_{i} \rho_{i}$ from before mass transfer in the calculation of $T_{i j}$ gives momentum conservation on transfer.

\subsubsection{Updates for Consistency}

So far, each time-step, we have updated each $\sigma_{i} \rho_{i}$ using the continuity equations, $U_{i}$ using the momentum equations, $\theta_{i}$ using the temperature equations and $\pi$ using the Helmholz equation which is a combination of the momentum and continuity equations and the equation of state. This is overspecified so to avoid inconsistencies growing (which leads to instability), $\pi$ is re-calculated from $\sigma_{i} \rho_{i}$ and $\theta_{i}$ at the end of every time-step using just the equation of state (14). The resulting changes to $\pi$ need to be small otherwise the updated $\pi$ would not solve the Helmholtz equation and acoustic modes would grow. Therefore $\rho_{i}$ must be solved with the same spatial discretisation as $\pi$ which, for best energy conservation (given 2nd order numerics) is centred linear differencing. This is why only $\sigma_{i}$, not $\sigma_{i} \rho_{i}$ is solved with a bounded advection scheme as described in section 3.2.2.

\subsubsection{Overview of the Solution Algorithm}

Each item below is carried out for all cells before moving on to the next item. This is crucial for the semi-implicit formulation.

1. We start by initialising all iterated variables at $k=0$ and $\ell=0$ to the values at time level $n$.

2. For $k=1,2$ :

(a) Solve for $\left(\sigma_{i} \rho_{i}\right)^{k}$ as described in section 3.2.2.

(b) Solve for $\theta_{i}^{k}$ as described in section 3.2.3.

(c) Update $\sigma_{i}^{k}$ as described in section 3.2.4.

(d) For $\ell=1,2$

i. Update each $U_{i}^{\prime \prime}$ using eqn (35) which consists of all of the terms of the momentum equation excluding the pressure gradient term and excluding transfer terms.

ii. Calculate the compressibility, $\Psi$, from eqn (38).

iii. Construct and solve the Helmholtz eqn (39) for $\pi^{\ell}$.

iv. Back substitute, adding the pressure gradient term to $U_{i}^{\prime \prime}$ to get $U_{i}^{\prime}$ using eqn (34).

v. Add the transfer terms to $U_{i}^{\prime}$ to get $U_{i}^{\ell}$ using eqn $(41,42)$.

vi. Calculate $\mathbf{u}_{i}^{\ell}$ from $U_{i}^{\ell}$ using appendix eqn (57).

3. For consistency, update $\Psi$ and $\pi$ at the end of each time-step from the equation of state (section 3.2.6). 


\section{Results}

No test cases exist for numerical solutions of the conditionally averaged Euler equations and so variations of the rising bubble test case (Bryan and Fritsch 2002) for the non-hydrostatic, compressible Euler equations are used. If the conditions in each fluid are initially identical, then the solution should evolve exactly like the single fluid equations with an additional advected tracer for the fluid fraction. This is therefore used as a first test of the numerical method. Tests are next formulated with different initial conditions in each fluid in order to check that the solution maintains stability, boundedness and some conservation properties. Finally, tests are created with fluid one initially empty and mass is transferred in. The solution should evolve exactly as the single fluid case because the initial conditions in fluid one, with no mass, should be irrelevant.

The dry, warm rising bubble test case of Bryan and Fritsch (2002) consists of a two dimensional vertical slice of height $10 \mathrm{~km}$ and width $20 \mathrm{~km}$ initially at rest with a surface pressure of $1000 \mathrm{mb}$, an initially uniform potential temperature of $300 \mathrm{~K}$. The initial pressure is in discrete hydrostatic balance with this uniform potential temperature. A warm perturbation:

$$
\theta^{\prime}=2 \cos ^{2} \frac{\pi L}{2}
$$

is added for $L<1$ where $L=\sqrt{\left(\frac{x-x_{c}}{x_{r}}\right)^{2}+\left(\frac{z-z_{c}}{z_{r}}\right)^{2}}, x_{c}=$ $10 \mathrm{~km}, z_{c}=2 \mathrm{~km}$ and $x_{r}=z_{r}=2 \mathrm{~km}$. $100 \mathrm{~m}$ grid spacing is used and for all simulations presented a time-step of $2 \mathrm{~s}$ is used. Regardless of the initial conditions and transfers between fluids, $\sigma$ should remain bounded between zero and one and the potential temperature should remain bounded between $300 \mathrm{~K}$ and $302 \mathrm{~K}$.

\subsection{Two Identical Fluids}

First the warm rising bubble of Bryan and Fritsch (2002) is simulated with the fluid divided into two with identical initial conditions in each fluid. No transfers or exchanges between fluids are used. Two different initial fluid fractions are used as shown at the top of figure 1 . These should not influence the evolution of other variables. The two initial $\sigma$ distributions are

$$
\begin{aligned}
& \text { symmetric: } \sigma_{1}= \begin{cases}1 & \text { if }\left|\mathbf{x}-\left(\begin{array}{l}
0 \\
2
\end{array}\right) \mathrm{km}\right|<2 \mathrm{~km} \\
0 & \text { otherwise }\end{cases} \\
& \text { asymmetric: } \sigma_{1}= \begin{cases}1 & \text { if }\left|\mathbf{x}-\left(\begin{array}{l}
2 \\
5
\end{array}\right) \mathrm{km}\right|<2 \mathrm{~km} \\
0 & \text { otherwise. }\end{cases}
\end{aligned}
$$

The distributions of $\sigma_{1}, \theta_{i}$ and the velocity in each fluid after $1000 \mathrm{~s}$ are shown at the bottom of figure $1 . \theta$ and the velocity have remained identical in each fluid and $\sigma$ has been advected by the flow without any undershoots or overshoots. The presence of the $\sigma$ field does not influence the evolution of the velocity or potential temperature in each fluid, as expected.

The stability of the model is demonstrated by plotting the total energy and the different types of energy in figure 2. The left hand side shows normalised kinetic, potential, internal and total energy changes for the model with a single fluid using van-Leer advection. The various energies are defined as:

$$
\begin{aligned}
\mathrm{KE} & =\frac{1}{2} \sum_{i} \sigma_{i} \rho_{i}\left|\mathbf{u}_{c i}\right|^{2} \\
\mathrm{PE} & =-\mathbf{g} \cdot \mathbf{x} \sum_{i} \sigma_{i} \rho_{i} \\
\mathrm{IE} & =c_{v} \pi \sum_{i} \sigma_{i} \rho_{i} \theta_{i} \\
\mathrm{E} & =\mathrm{KE}+\mathrm{PE}+\mathrm{IE}
\end{aligned}
$$

and totals are calculated by integrating over space. The normalisation and calculation of changes is calculated for energy $\mathrm{XE}$ as:

$$
\widetilde{\mathrm{XE}}=\frac{\mathrm{XE}-\mathrm{XE}(t=0)}{\mathrm{E}(t=0)} .
$$

The dashed lines in figure 2 show negative values and the solid lines show positive values. In the first part of the simulation, the single fluid simulation shows internal and potential energy oscillating in phase with each other, showing nearly conservative transfers between internal and potential energy. Throughout the simulation the kinetic energy increases as the rising bubble accelerates while the total energy decreases monotonically due to stable, dissipative nature of the model. Part of this dissipation is due to the dissipative advection of velocity (linear-upwind) and potential temperature (van-Leer). A simulation is also run using centred, linear differencing for advection and the total energy is shown in the right hand of figure 2. This simulation loses energy more slowly and the energy loss is no longer monotonic. The temperature field is noisy with spurious overshoots and undershoots of temperature (not shown).

The accuracy of the energy conservation in figure 2 appears to be good partly because the energy changes are divided by a large number - the total initial energy, including unavailable energy. The initial potential energy, which is mostly unavailable, is 31,444 Joules and the initial internal energy is 141,446 Joules, making the total initial energy 172,891 Joules. A fairer accuracy estimate might be to normalise with the available potential energy. This has not been calculated. Instead we could compare with the energy of the initial warm bubble. The warm bubble contains 24.5 Joules of additional internal energy in comparison to the stably stratified state. If we were to normalise the energy changes with this value then they would be 7,057 times bigger, making a normalised change of $10^{-6}$ close to a change of 0.01 .

The total energy for the simulations with two identical fluids with symmetric and asymmetric distributions of $\sigma$ are shown in figure 2. This confirms that the presence of more than one identical fluid does not influence the energy conservation. In fact the solutions with two identical fluids are identical to the solution with one fluid to within machine precision.

\subsection{Different Initial Conditions in each Fluid}

Once each fluid has different properties, the behaviour of the solutions changes and stable solutions may not exist. The total solution is close to divergence free because compressibility is small in this low Mach number regime. However with only one pressure to control the divergence in two fluids, the divergence in each fluid can be large. We will therefore initialise the model to force different velocities and hence divergence in each fluid. We do not have an analytic solution for this case but we seek stable solutions and we test energy conservation since energy is conserved in the continuous equations in the absence of transfers between the fluids.

In order to simulate two fluids with different properties occupying the same location, we set $\sigma_{1}=\frac{1}{2}$ in a circle with warm air only in fluid 1 and initially stationary flow in each fluid:

$$
\begin{aligned}
\sigma_{1} & =\left\{\begin{array}{cc}
\frac{1}{2} & \text { if }\left|\mathbf{x}-\left(\begin{array}{c}
0 \\
2
\end{array}\right) \mathrm{km}\right|<2 \mathrm{~km} \\
0 & \text { otherwise }
\end{array}\right. \\
\sigma_{0} & =1-\sigma_{1} \\
\theta_{0} & =300 \mathrm{~K} \\
\theta_{1} & =300 \mathrm{~K}+\theta^{\prime}
\end{aligned}
$$

We assume no mass flux and no drag between fluids. The initial conditions for $\sigma_{1}$ and $\theta_{1}$ are shown in figure 3 along with the 
Initial $\sigma_{1}$ (shaded) and initial $\theta_{i}$ (contoured every $0.2 \mathrm{~K}$ )

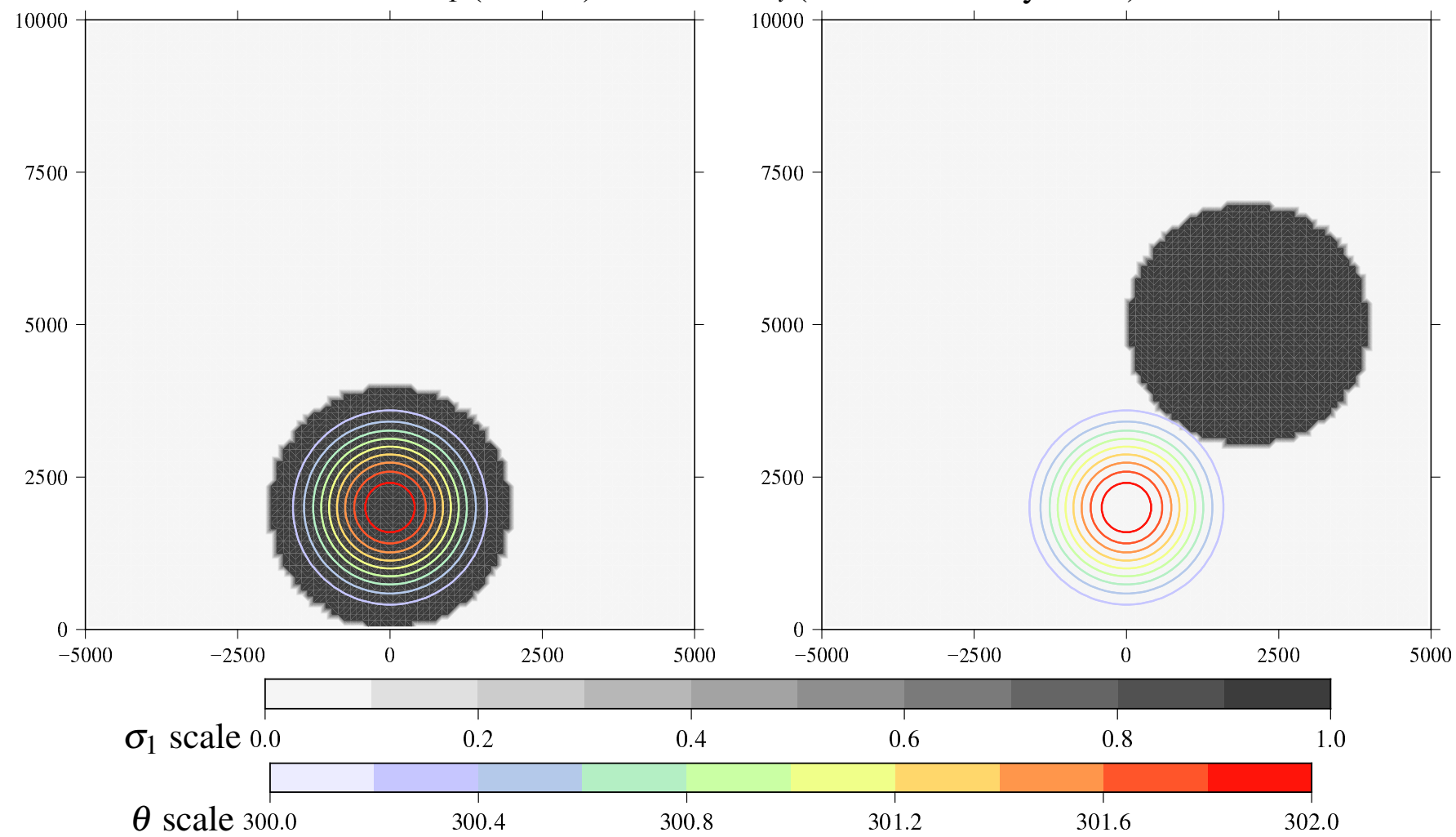

$\sigma_{1}, \theta_{i}$ and $\mathbf{u}_{i}$ after $1000 \mathrm{~s}$ (results identical in each fluid)
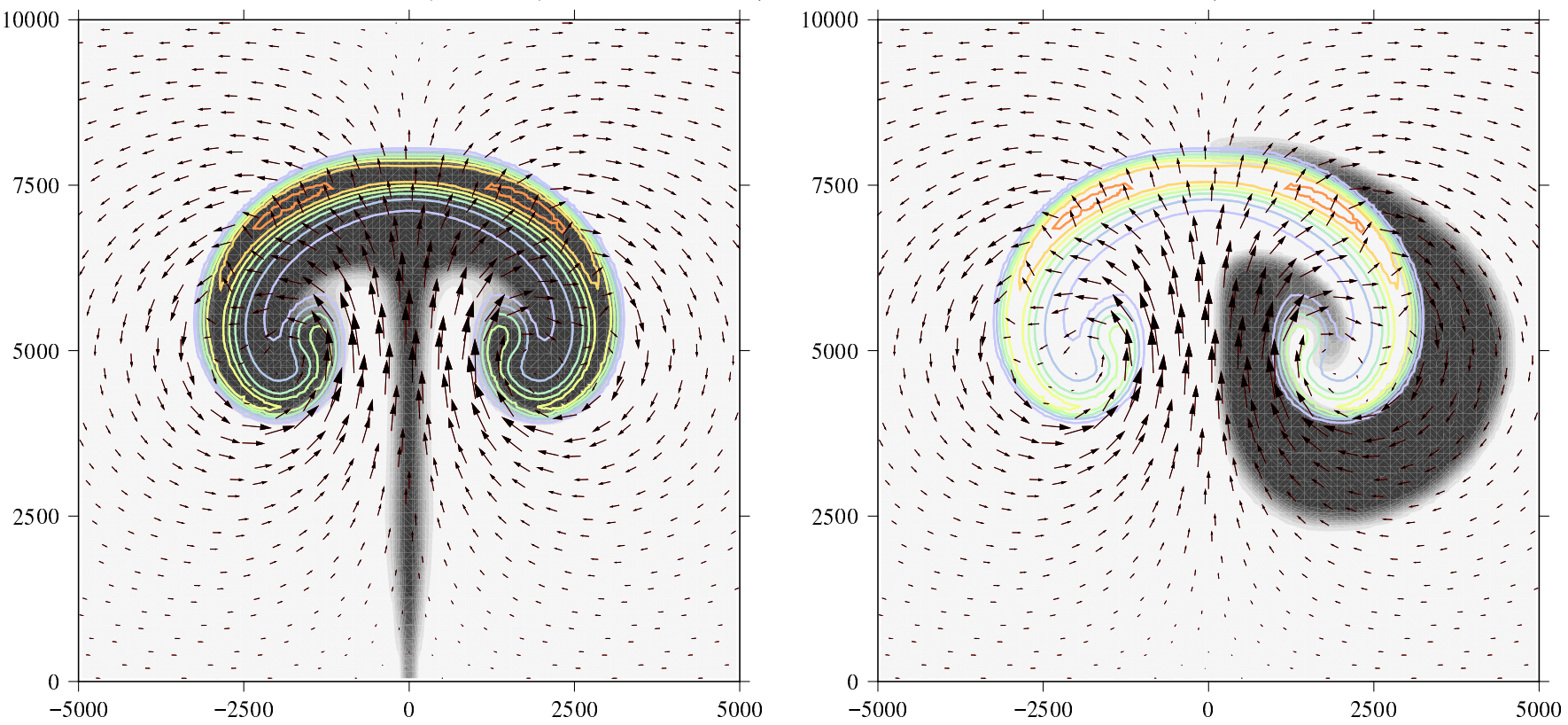

Figure 1. Initial fluid fraction, $\sigma_{1}$ and $\theta_{i}$ (top) for simulations with identical properties in each fluid and properties after $1000 \mathrm{~s}$ (bottom). The vectors and contours for $\theta_{i}$ and $\mathbf{u}_{i}$ for each fluid are identical. The full domain extends between $x=-10^{4} \mathrm{~m}$ and $x=10^{4} \mathrm{~m}$

solutions after 100,200 and 290 s. $\theta_{0}$ remains identically $300 \mathrm{~K}$ throughout the simulation, as expected.

The buoyancy perturbation in fluid one makes fluid one rise which raises the pressure above the bubble and forces fluid zero downwards. Consequently total divergence is controlled but the divergence in each fluid grows and hence the velocities become large. The advection scheme is only bounded for Courant numbers less than 0.5 . At $276 \mathrm{~s}$, the mean Courant number becomes larger than 0.5 and oscillations grow in $\sigma_{1}$. The solution diverges at $t=296 \mathrm{~s}$. A stable simulation could be maintained for a little longer by using a smaller time-step or by treating advection implicitly but the PDEs that we are solving are unstable (Stewart and Wendroff 1984) so numerics cannot be used to maintain stability indefinitely.

\subsubsection{Coupling the two fluids with drag or diffusion}

We first add drag between the fluids but no mass transfer. The drag takes the form described in section 2.1.1 with length scales $r_{\min }=1 \mathrm{~m}$ (a minimum is needed to avoid division by zero) and $r_{\max }=2000 \mathrm{~m}$. A large value of $r_{\max }$ has been chosen so that the drag is low where neither $\sigma_{i}$ is vanishing which allows some variation of velocity between fluids. The results at $t=1000 \mathrm{~s}$ are shown in figure $4 . \theta_{0}$ remains identically $300 \mathrm{~K}$ throughout. The drag has stabilised the solution and the two fluid velocities (in black and red) are not identical.

Next the fluids are coupled by adding diffusion between the fluids (but no drag) as described in section 2.1.2. This is similar to convective entrainment and detrainment due to turbulence. 
1 fluid, van-Leer advetion

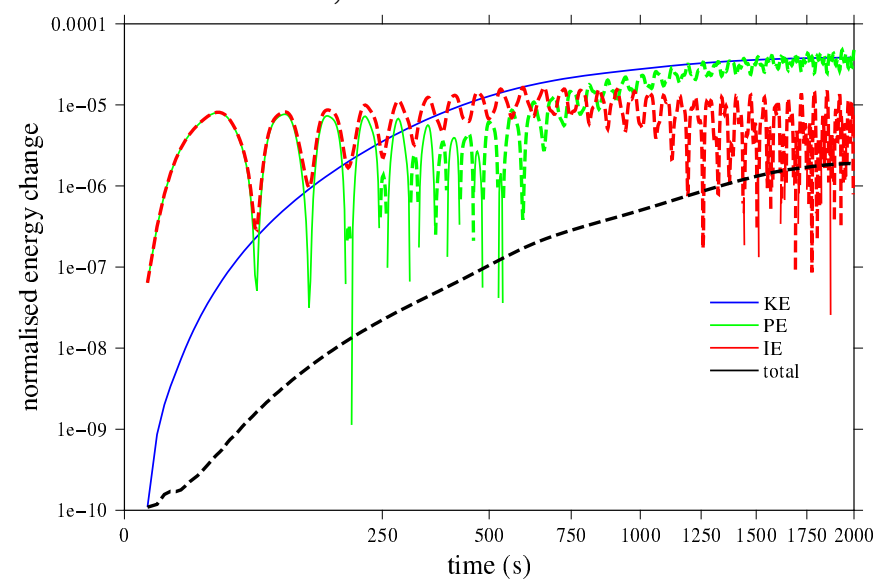

Total energy for various simulations

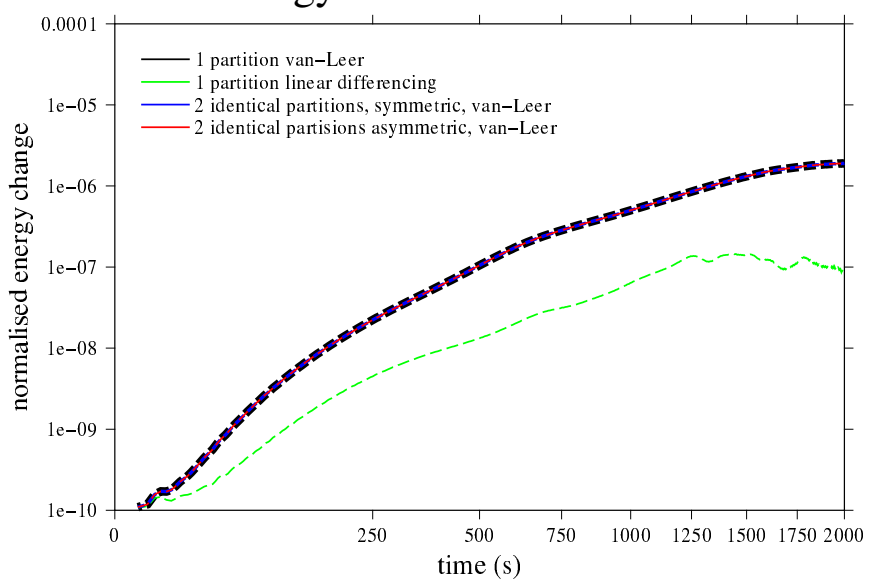

Figure 2. Normalised changes in kinetic, potential, internal and total energy for the rising bubble test case for the model with a single fluid and the normalised total energy change for models with one fluid with different advection schemes and for a model with two identical fluids and no mass transfer. Solid lines show positive changes and dashed lines show negative changes. The $\mathrm{x}$-axis is on a square root scale to emphasise the changes that occur early in the simulations.

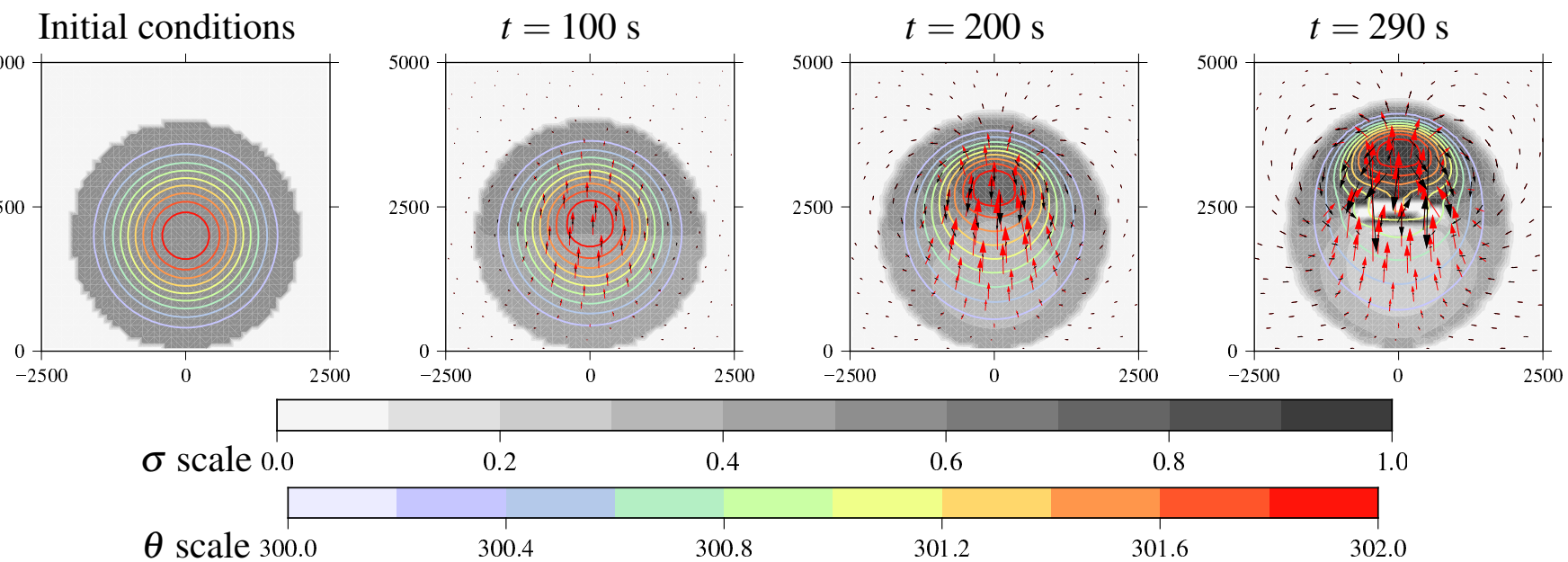

Figure 3. Zoomed in initial conditions and results of a simulation with a buoyant perturbation in fluid 1 and no mass transfer. $\sigma_{1}$ is shaded, $\theta_{1}$ is contoured every $0.2 \mathrm{~K}$, $\mathbf{u}_{0}$ is shown by black vectors and $\mathbf{u}_{1}$ by red vectors. The full domain extends between $x=-10^{4} \mathrm{~m}$ and $x=10^{4} \mathrm{~m}$ and between $z=0$ and $z=10^{4} \mathrm{~m}$.

The results using a diffusion coefficient of $K_{\sigma}=200 \mathrm{~m}^{2} \mathrm{~s}^{-1}$ are shown in figure 4 . The diffusion is treated explicitly and this diffusion coefficient is well below the stability limit. Once mass is transferred, the peaks in $\sigma_{1}$ reduce in comparison to the simulation with drag between the fluids. Temperature and momentum are transferred with the mass so the temperature in fluid 1 no longer remains $300 \mathrm{~K}$ and the temperature in fluid 0 reduces.

The total energy changes for all of the simulations with warm air in a diffuse fluid 1 are shown in the bottom right of figure 4. The energy diverges for the unstable case with no transfers. The other simulations are stabilised either by mass transfers or by momentum transfer (drag). Both of these destroy kinetic energy and so we expect to see the energy decrease monotonically for the stabilised simulations. The simulation with the low diffusion coefficient $\left(K_{\sigma}=200 \mathrm{~m}^{2} \mathrm{~s}^{-1}\right)$ stabilises the model after around $350 \mathrm{~s}$ but this looks unreliable as energy increases before this point. A larger diffusion coefficient, $\left(K_{\sigma}=800 \mathrm{~m}^{2} \mathrm{~s}^{-1}\right)$ stabilises the model more effectively. (The stability limit is $K_{\sigma} \Delta t / \Delta x^{2}<$ $\frac{1}{2}$ so for $\Delta t=2 \mathrm{~s}$ and $\Delta x=100 \mathrm{~m}$ the stability limit is $K_{\sigma}=$ $2500 \mathrm{~m}^{2} \mathrm{~s}^{-1}$.) The simulation is also stabilised by using drag between the fluids. The low drag coefficient of $C_{D}=1, r_{\min }=$ $1 \mathrm{~m}$ and $r_{\max }=2000 \mathrm{~m}$ leads to more rapid energy loss than any of the other effective stabilisation methods. A high drag coefficient $\left(C_{D}=10^{6}\right)$ is also used to check the stability of the implicit treatment of drag. It is not immediately obvious why the high drag coefficient would lead to less energy loss when we usually think of drag as removing kinetic energy. With high drag between the fluids, kinetic energy is more evenly spread between the fluids and lower velocities and lower shear could lead to less kinetic energy dissipation by the dissipative advection.

\subsection{Transfers Between Fluids to mimic convection parameterisation}

We seek to demonstrate that the numerical method is stable in the presence of large transfers between fluids and we choose transfer terms that lead to warm rising air in fluid one and stable and descending air in fluid zero. We examine stability, energy conservation and compare with the single fluid case. These simulations start with no fluid one (all the mass initially in fluid zero). Whenever mass is transferred into fluid one it takes its properties with it so the solution should be identical to the single fluid case and energy should be conserved. In order to maintain stability, diffusion between fluids of $K_{\sigma}=100 \mathrm{~m}^{2} \mathrm{~s}^{-1}$ and drag with coefficient of $C_{D}=1 / 2, r_{\min }=1 \mathrm{~m}$ and $r_{\max }=2000 \mathrm{~m}$ are used.

\subsubsection{Transfers based on buoyancy perturbations}

We test the numerical solution using the mass transfer terms associated with buoyancy perturbations from eqns $(21,22)$. To test that warm air is transferred from fluid zero to fluid one, we initialise the simulation with $\sigma_{0}=0$ everywhere and the warm perturbation in fluid zero only. The solutions for $\sigma_{1}, \theta_{0,1}$ and $\mathbf{u}_{0,1}$ after $1000 \mathrm{~s}$ are shown in figure 5 using diffusivities 


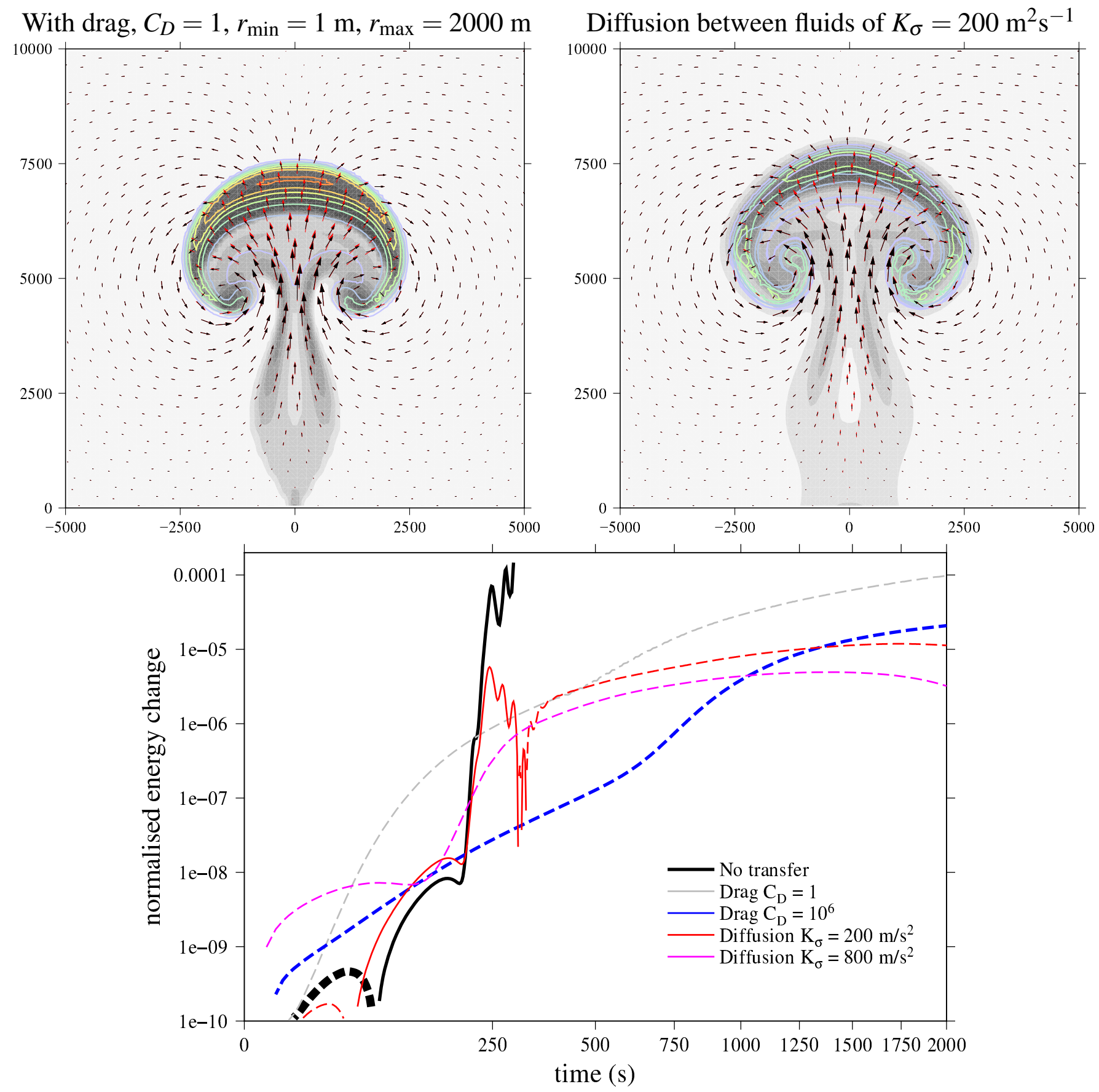

Figure 4. Results at $t=1000 \mathrm{~s}$ of simulations with stabilisation with a buoyant perturbation in fluid $1 . \sigma_{1}$ is shaded, $\theta_{1}$ and $\theta_{2}$ are contoured every $0.2 \mathrm{~K}$, $\mathbf{u}_{0}$ is shown by black vectors and $\mathbf{u}_{1}$ by red vectors. Initially $\sigma=\frac{1}{2}$ in a circle near the ground (as in fig 3 ). The time-series shows the normalised energy changes. Dashed lines show negative changes. The $\mathrm{x}$-axis is on a square root scale to emphasise the changes that occur early in the simulations.

$K_{\theta}=10^{6} \mathrm{~m}^{2} \mathrm{~s}^{-1}$ (top left) and $K_{\theta}=10^{5} \mathrm{~m}^{2} \mathrm{~s}^{-1}$ (bottom left). Figure 5 confirms that $\theta_{i}$ and $\mathbf{u}_{i}$ are similar in each fluid and also similar to the solutions in figure 1 . Using $K_{\theta}=10^{6} \mathrm{~m}^{2} \mathrm{~s}^{-1}$, warm fluid is completely transferred to fluid one whereas using $K_{\theta}=10^{5} \mathrm{~m}^{2} \mathrm{~s}^{-1}$, the transfer is partial.

The $\ell_{2}$ errors shown in figure 5 show the root mean square difference between the single fluid $\theta$ and the mean $\theta$ across all partitions $\left(\sum_{i} \sigma_{i} \rho_{i} \theta_{i} / \sum_{i} \sigma_{i} \rho_{i}\right)$ normalised by the root mean square single fluid $\theta$. Fluid one is initialised with no mass and without a warm bubble. The zero mass in fluid one means that once mass is transferred to fluid one it should have identical properties to fluid zero. This does not happen exactly; the RMS errors are low but not zero. These RMS errors can be compared with the RMS differences between the simulations initialised with identical properties in both fluids and the single fluid in figure 1 . These have RMS differences of $7.7 \times 10^{-14}$. Therefore the simulation of a zero mass fluid with different initial conditions is introducing numerical error although these errors are not obvious when comparing the results of figure 5 with those in figure 1 by eye.

The changes in energy for both solutions with mass transfer based on buoyancy perturbations are shown in figure 6. The energy loss is very similar to the single fluid case (figure 2).

\subsubsection{Transfers based on horizontal divergence and vertical velocity}

The results of the simulation using mass transfer based on horizontal divergence and vertical velocity from eqns $(23,24)$ are shown on the top right of figure 5. Again, since fluid one is initially empty, its initial properties should not influence the final solution. Instead, properties are transferred from fluid zero and the two fluids evolve in the same way. The solutions are very similar to the single fluid simulation, with normalised RMS differences 

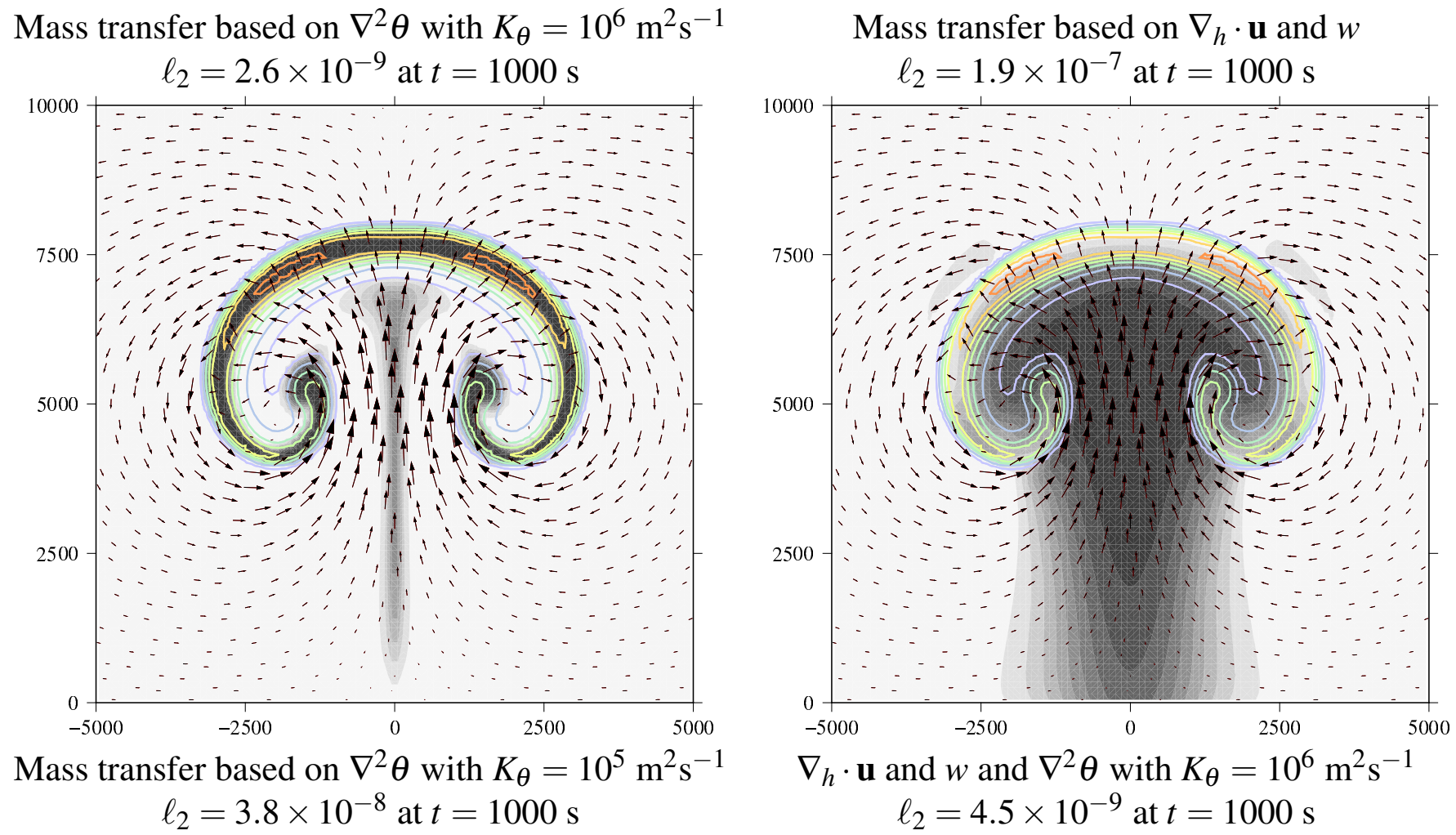

Mass transfer based on $\nabla^{2} \theta$ with $K_{\theta}=10^{5} \mathrm{~m}^{2} \mathrm{~s}^{-1}$
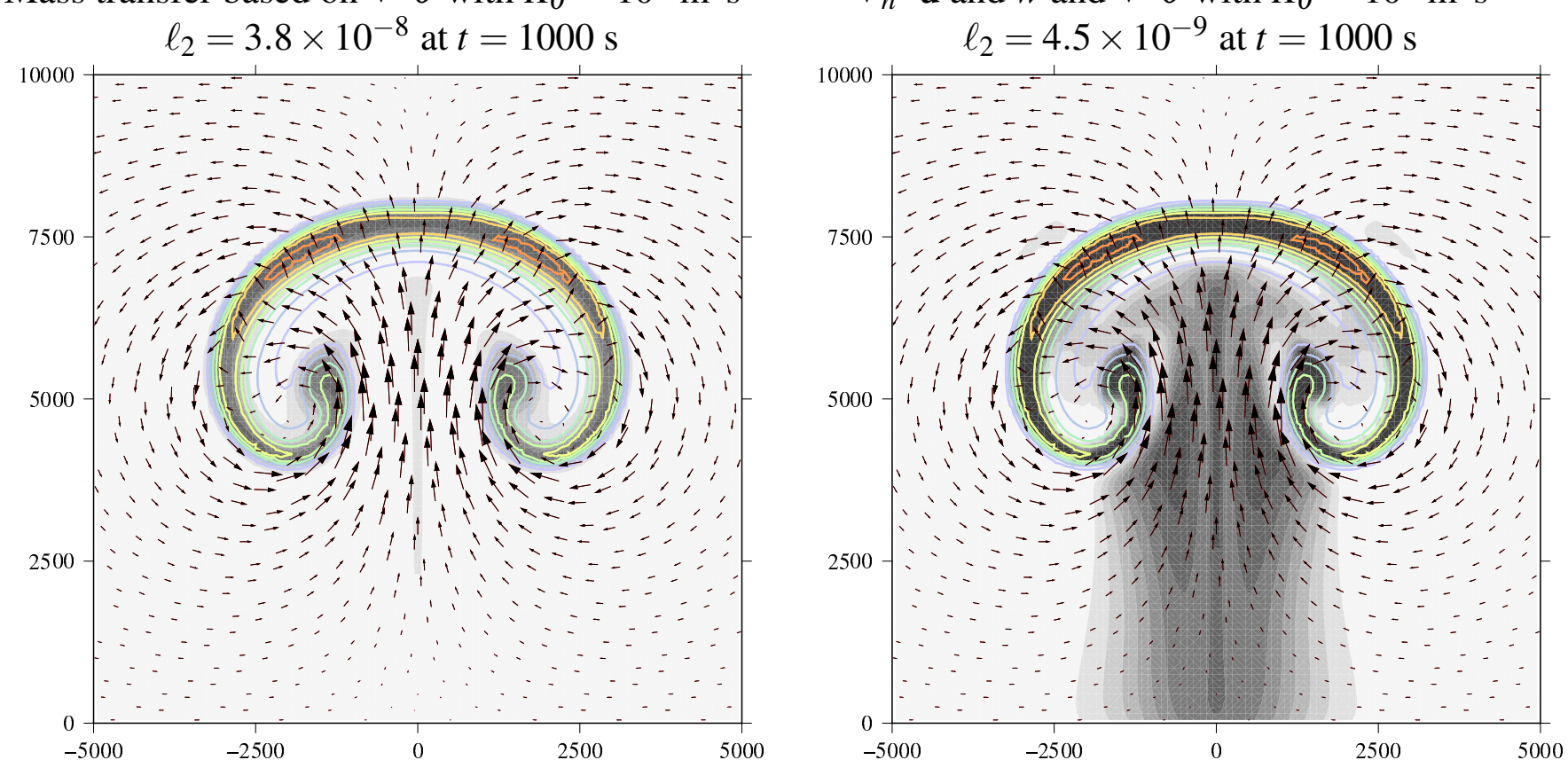

Figure 5. Rising bubble solutions of $\sigma_{1}, \theta_{0,1}$ and $\mathbf{u}_{0,1}$ after $1000 \mathrm{~s}$ with mass transfers based on $K_{\theta} \nabla^{2} \theta / \theta$ and based on $\nabla_{h} \cdot \mathbf{u}$ and $\mathbf{u} \cdot \mathbf{g}$. All simulations use $K_{\sigma}=100 \mathrm{~m}^{2} \mathrm{~s}^{-1}$ and drag with coefficient of $C_{D}=1 / 2, r_{\min }=1 \mathrm{~m}$ and $r_{\max }=2000 \mathrm{~m}$.

The $\ell_{2}$ error norms are the normalised root mean square difference between the single fluid $\theta$ and the mean $\theta$ across all partitions $\left(\sum_{i} \sigma_{i} \rho_{i} \theta_{i} / \sum_{i} \sigma_{i} \rho_{i}\right)$.

of $1.9 \times 10^{-7}$ which again is not big enough to visually see differences between the results in figures 5 and 1 . Using mass transfer based on horizontal divergence leads to larger differences from the single fluid case but more mass is transferred so this is not a disadvantage of mass transfer based on horizontal divergence. However transfer based on horizontal divergence leads to transfer of the fluid that is behind the warm air and not the warm air itself. The warm air actually expands horizontally and so is not transferred. Using mass transfer based on horizontal divergence, the drop in energy is again very similar to the single fluid case (comparing figures 2 and 6).

\subsubsection{Transfers based on buoyancy perturbations, horizontal divergence and vertical velocity}

Finally we use both types of mass transfer in the simulation shown in the bottom right of figure 5. One might expect the fluid transferred to be the sum of the fluids transferred based on both processes separately but this is not the case because of inconsistencies between the two transfer processes. For example, $\nabla^{2} \theta$ is positive behind the warm anomaly so fluid is transferred back to fluid 1, despite the horizontal convergence and rising air. It does not appear useful to use both types of transfer.

\section{Summary, Conclusions and Further Work}

A stable numerical method is presented for solving the conditionally averaged equations of motion for representing 


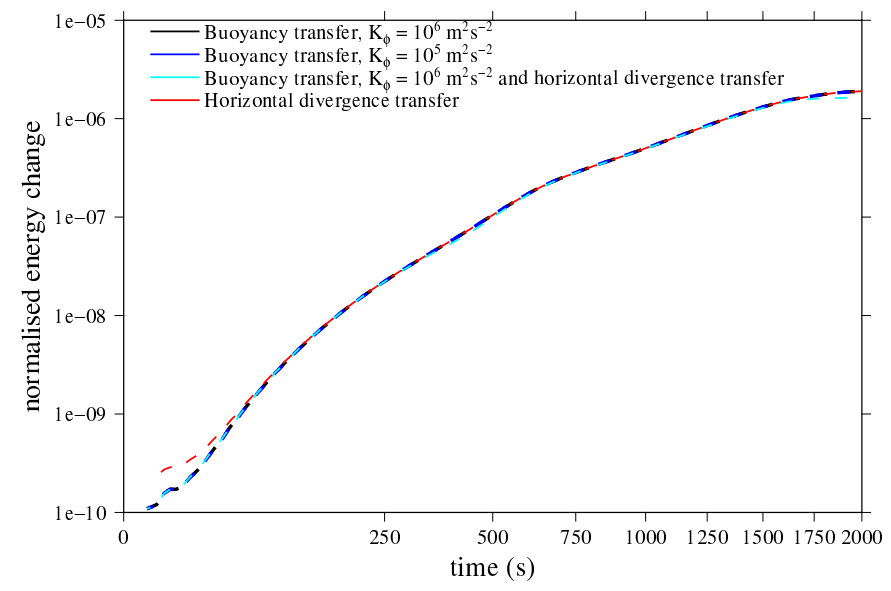

Figure 6. Normalised energy changes for the rising bubble solutions with mass transfer based on $K_{\theta} \nabla^{2} \theta / \theta$ and based on $\nabla_{h} \cdot \mathbf{u}$ and $\mathbf{u} \cdot \mathrm{g}$. Dashed lines show negative changes (all changes are negative). The $\mathrm{x}$-axis is on a square root scale to emphasise the changes that occur early in the simulations.

atmospheric convection with two fluids, one to represent stable, environment air and the other to represent buoyant plumes. This builds on traditional mass flux schemes by solving equations for mass, temperature and momentum both inside and outside the plumes and so net mass transfer by convection can be represented. Our numerical method would also be suitable for the similar extended EDMF scheme (Tan et al. 2018) and would allow net mass transport by convection. Transfers of mass between the fluids are proposed for two purposes:

1. The conditionally averaged equations with a single pressure are ill-posed so transfer terms are formulated to represent drag and mass transfer between fluids to ensure that the different fluids do not diverge.

2. Transfer terms are proposed so that well resolved convection is transferred to the buoyant fluid and stable or sinking air is transferred to the stable fluid. These are based on buoyancy anomalies (measured by the Laplacian of $\theta$ ) and based on horizontal divergence.

The transfer terms are applied explicitly to the individual partition continuity equations (the transport equations for $\sigma_{i} \rho_{i}$ ) and limited to avoid negative mass in each fluid. These mass transfer terms also appear in the temperature and momentum equations since the transferred mass takes its other properties with it. In these equations the transfer terms are treated implicitly as they can be very large. The numerical treatment of these transfer terms ensures boundedness and mass, momentum and internal energy conservation on transfer.

A semi-implicit finite volume method is used to solve the equations of motion in advective form which ensures boundedness of the fluid fraction, $\sigma_{i}$, and removes time-step restrictions based on the speed of sound. This aspect of the solution entails substituting momentum equations of both (or all) fluids into the continuity equation rather than just the mean flow momentum equation as is done in semi-implicit weather forecast models. Without this numerical treatment, net mass transport by convection would trigger acoustic waves that are not handled by the implicit part of the model which would lead to instability for moderate time-steps.

The results presented in this paper use explicit Eulerian advection schemes and have advective Courant number limits of 0.5 . This Courant number restriction could be raised with the use of semi-Lagrangian or implicit advection. This could be important for large updrafts in plumes.

Results are presented of a well resolved rising warm bubble simulated with the two fluid equations. Without transfer terms the model is unstable since divergence in each fluid is not controlled. The model is stabilised by adding either lateral entrainment as diffusion of mass between the fluids or by adding drag between the fluids. Transfer terms are also included to move the warm rising air from the stable fluid to the buoyant fluid. The model loses energy slowly due mainly to dissipative advection. The transfer terms are implemented for stability and so that internal energy is conserved.

This paper has not answered the question of how much stabilisation of the conditionally averaged equations is necessary, just that two different techniques can stabilise a model of the conditionally averaged equations. Numerical analysis to find the minimum necessary stabilisation will be the subject of future work.

The formulation of transfer terms in order to represent sub-grid convection is also the subject of future work. These transfer terms should depend on sub-grid variability of the primitive variables in each fluid. For representing unresolved flow, parameterisations for sub-filter-scale fluxes will also be essential.

\section{Acknowledgements}

Many thanks to Peter Clark, John Thuburn, Chris Holloway and Dan Shipway for valuable discussions and proof reading. Thanks to the NERC/Met Office Paracon project. We acknowledge funding from the RevCon Paracon project NE/N013743/1.

\section{References}

A. Arakawa and C.-M. Wu. A unified representation of deep moist convection in numerical modeling of the atmosphere. part I. J. of Atmos Sci., 70:19771992, 2013.

G. Bryan and J. Fritsch. A benchmark simulation for moist nonhydrostatic numerical models. Mon. Wea. Rev., 130(12):2917-2928, 2002.

W. de Rooy, P. Bechtold, K. Froehlich, C. Hohenegger, H. Jonker, D. Mironov, A. Siebesma, J. Teixeira, and J.-I. Yano. Entrainment and detrainment in cumulus convection: an overview. Quart. J. Roy. Meteor. Soc., 139(670, A): 1-19, 2013.

C. Dopazo. On conditioned averages for intermittent turbulent flows. J. Fluid Mechanics, 81:433-438, 71977.

L. Gerard and J.-F. Geleyn. Evolution of a subgrid deep convection parametrization in a limited-area model with increasing resolution. Quart. J. Roy. Meteor. Soc., 131:2293-2312, 2005.

D. Gregory and P. Rowntree. A mass flux convection scheme with representation of cloud ensemble charateristics and stability-dependent closure. Mon. Wea. Rev., 118:1483-1506, 1990.

A. Guelfi, D. Bestion, M. Boucker, P. Boudier, P. Fillion, M. Grandotto, J.-M. Hérard, E. Hervieu, and P. Péturaud. NEPTUNE: A new software platform for advanced nuclear thermal hydraulics. Nuclear Science and Engineering, 156:281-324, 2007.

C. Holloway, J. Petch, R. Beare, P. Bechtold, G. Craig, S. Derbyshire, L. Donner, P. Field, S. Gray, J. Marsham, D. Parker, R. Plant, N. Roberts, D. Schultz, A. Stirling, and S. Woolnough. Understanding and representing atmospheric convection across scales: recommendations from the meeting held at Dartington Hall, Devon, UK, 28-30 January 2013. Atmos. Sci. Lett., 15(4):348-353, 2014.

D. Holm and B. Kupershmidt. Multipressure regularizatoin for multiphase flow. Physics Letters, 106A(4):165-168, 1984.

J. Kain and J. Fritsch. A one-dimensional entraining/detraining plume model and its application in convective parameteriztion. J. of Atmos Sci., 47(23): 2784-2802, 1990.

V. Kuell and A. Bott. A hybrid convection scheme for use in non-hydrostatic numerical weather prediction models. Meteorologische Zeitschrift, 2008.

V. Kuell, A. Gassmann, and A. Bott. Towards a new hybrid cumulus parametrization scheme for use in non-hydrostatic weather prediction models. Quart. J. Roy. Meteor. Soc., 2007.

M. Lance and J. Bataille. Turbulence in the liquid phase of a uniform bubbly air-water flow. Journal of Fluid Mechanics, 222:95-118, 11991.

H. Lean, P. Clark, M. Dixon, N. Roberts, A. Fitch, R. Forbes, and C. Halliwell. Characteristics of high-resolution versions of the Met Office Unified Model for forecasting convection over the United Kingdom. Mon. Wea. Rev., 136: 3408-3424, 2008.

S. Park. A unified convection scheme (UNICORN). Part I: formulation. J. of Atmos Sci., 71(11):3902-3930, 2014. 
I. Roghair, Y. Lau, N. Deen, H. Slagter, M. Baltussen, M. Van Sint Annaland, and J. Kuipers. On the drag force of bubbles in bubble swarms at intermediate and high Reynolds numbers. Chemical Engineering Science, 66(14):3204-3211, 2011

D. Romps and A. Charn. Sticky thermals: Evidence for a dominant balance between buoyancy and drag in cloud updrafts. J. of Atmos Sci., 72(8):28902901, 2015.

S. Sherwood, M. Alexander, A. Brown, N. McFarlane, E. Gerber, G. Feingold, A. Scaife, and W. Grabowski. Climate Science for Serving Society, chapte Climate Processes: Clouds, Aerosols and Dynamics. Springer, Dordrecht, 2013.

A. Simmons, D. Burridge, M. Jarraud, C. Girard, and W. Wergen. The ECMWF medium-range prediction models: development of the numerical formulations and the impact of increased resolution. Meteorol. Atmos. Phys., 1989.

J. Simpson and V. Wiggert. Models of precipitating cumulus towers. Mon Wea. Rev., 97(7):471-489, 1969.

S. Solomon, D. Qin, M. Manning, Z. Chen, M. Marquis, K. Averyt, M. Tignor, and H. Miller. IPCC, 2007: Climate change 2007: The physical science basis. Technical report, Intergovernmental Panel on Climate Change, 2007. Contribution of Working Group 1 to the Fourth Assessment Report.

G. Stephens, T. L'Ecuyer, R. Forbes, A. Gettelmen, J.-C. Golaz, A. BodasSalcedo, K. Suzuki, P. Gabriel, and J. Haynes. Dreary state of precipitation in global models. J. Geophys. Res. - Atmospheres, 115(D24), 2010.

H. Stewart and B. Wendroff. Two-phase flow: Models and methods. J. Comput. Phys., 56(3):363-409, 1984.

Z. Tan, C. Kaul, K. Pressel, Y. Cohen, T. Schneider, and J. Teixeira. An extended eddy-diffusivity mass-flux scheme for unified representation of subgrid-scale turbulence and convection. JAMES, 10(3):770-800, 2018.

J. Thuburn, H. Weller, G. K. Vallis, R. J. Beare, and M. Whitall. A framework for convection and boundary layer parameterization derived from conditional filtering. J. of Atmos Sci., 75(3):965-981, 2018.

J. Thuburn, G. Efstathiou, and R. Beare. A two-fluid single-column model of the dry convective boundary layer. Quart. J. Roy. Meteor. Soc., in prep, 2019.

H. Weller and A. Shahrokhi. Curl-free pressure gradients over orography in a solution of the fully compressible Euler equations with implicit treatment of acoustic and gravity waves. Mon. Wea. Rev., 142(12):4439-4457, 2014.

H. Weller, S.-J. Lock, and N. Wood. Runge-Kutta IMEX schemes for the horizontally explicit/vertically implicit (HEVI) solution of wave equations J. Comput. Phys., 252:365-381, 2013.

N. Wood, A. Staniforth, A. White, T. Allen, M. Diamantakis, M. Gross, T. Melvin, C. Smith, S. Vosper, M. Zerroukat, and J. Thuburn. An inherently mass-conserving semi-implicit semi-lagrangian discretisation of the deepatmosphere global nonhydrostatic equations. Quart. J. Roy. Meteor. Soc., 140:1505-1520, 2014.

J. Yano and R. Plant. Finite departure from convective quasi-equilibrium periodic cycle and discharge-recharge mechanism. Quart. J. Roy. Meteor Soc., 138:626-637, 2012.

\section{A. Spatial Discretisation}

The spatial discretisation used to solve the multi-fluid Euler equations is similar to Weller and Shahrokhi (2014) which was used for single fluid equations. None of the spatial discretisation described is specific for multi-fluid equations. The spatial discretisation is general for arbitrarily structured meshes (for example including hexagonal prisms, cut cells and refinement) but the meshes used in this study are all fully structured.

\section{A.1. Reconstruction of velocity fields at cell centres and faces from face normals}

The model uses a C-grid so normal components of the velocity (volume fluxes) are stored at cell faces. Full velocity fields are needed in the non-linear advection term, $\mathbf{u} \cdot \nabla \mathbf{u}$, of the momentum equation (16). The volume flux across each face is:

$$
U_{i}=\mathbf{u}_{f i} \cdot \mathbf{S}_{f}
$$

where $\mathbf{u}_{f}$ is the velocity at the face and $\mathbf{S}_{f}$ is the face area vector. The face velocity is interpolated from the cell centre velocity using linear interpolation:

$$
\mathbf{u}_{f i}=\lambda \mathbf{u}_{c i}+(1-\lambda) \mathbf{u}_{N i}
$$

where $\mathbf{u}_{c i}$ is the cell centre velocity of the cell that owns face $f$, $\mathbf{u}_{N i}$ is the cell centre velocity of the cell on the other side of face $f$ and $\lambda$ is the linear interpolation weight. The face area vector, $\mathbf{S}_{f}$ is normal to the face, has the area of the face and points from the owner cell to the neighbour cell. The cell centre velocity is reconstructed from surrounding values of $U_{i}$ using the standard OpenFOAM fvc: : reconstruct:

$$
\mathbf{u}_{c i}=\left(\sum_{f \in c} \hat{\mathbf{S}}_{f} \mathbf{S}_{f}^{T}\right)^{-1} \sum_{f \in c} U_{i} \hat{\mathbf{S}}_{f}
$$

where the hat denotes the unit vector and the notation $f \in c$ means all the faces, $f$ of cell $c$. Note that $\sum_{f \in c} \hat{\mathbf{S}}_{f} \mathbf{S}_{f}^{T}$ is a tensor defined on each cell that depends only on the mesh and its inverse multiplies the vector $\sum_{f \in c} U_{i} \hat{\mathbf{S}}_{f}$ for each cell.

This reconstruction is first-order accurate on arbitrary meshes and second-order accurate on uniform structured meshes. It simplifies to simple averaging of nearest neighbours on a uniform structured mesh of hexahedra.

\section{A.2. Non-Linear Advection}

This section is again concerned with the discretisation of the non-linear advection term, $\mathbf{u} \cdot \nabla \mathbf{u}$, of the momentum equation (16). The non-linear advection described is not optimal but yields satisfactory solutions using existing OpenFOAM operators to implement a C-grid on an unstructured mesh.

The conditionally averaged Euler equations are solved in advective form so that they are defined where $\sigma_{i}=0$ and so that a bounded advection scheme can be applied to $\sigma_{i}$. The finite volume technique most naturally lends itself to solving equations in flux form rather than advective form and so the non-linear advection term of the momentum equation is calculated as:

$$
\mathbf{u}_{i} \cdot \nabla \mathbf{u}_{i}=\nabla \cdot\left(\mathbf{u}_{i}^{T} \mathbf{u}_{i}\right)-\mathbf{u}_{i} \nabla \cdot \mathbf{u}_{i}
$$

This quantity is calculated at cell centres and then linearly interpolated onto faces. Both terms use Gauss's divergence theorem:

$$
\mathbf{u}_{i} \cdot \nabla \mathbf{u}_{i} \approx \frac{1}{V}\left(\sum_{f \in c} \mathbf{u}_{a i} U_{i}-\mathbf{u}_{c i} \sum_{f \in c} U_{i}\right)
$$

where $V$ is the cell volume and $\mathbf{u}_{a i}$ is the velocity interpolated from cell centres to faces using the OpenFOAM (second-order) linear upwind advection scheme. The prognostic variable is $U_{i}$ so the non-linear advection term from eqn (59) is linearly interpolated onto faces and then the dot product is taken with $\mathbf{S}_{f}$. This advection is not bounded and requires more interpolations and reconstructions than are usually used for C-grid advection of velocity. It is used because it makes implementation of Cgrid advection straightforward in OpenFOAM. The linear-upwind advection can be replaced by linear advection which gives better energy conservation but the results are a bit noisy (not shown).

\section{A.2.1. Pressure Gradient including the $\theta_{i}$ pre-factor}

The pressure gradient term, $c_{p} \theta_{i} \nabla \pi$, of the momentum equation (16) needs to be calculated on faces in the normal direction to the face. Since we assume an orthogonal mesh this can simply be calculated as the difference in pressure between the cells either 
side of the face:

$$
c_{p} \theta_{i} \nabla \pi \cdot \mathbf{S}_{f}=c_{p} \theta_{f i} \nabla_{S} \pi \approx c_{p} \theta_{f i} \frac{\pi_{c}-\pi_{N}}{\delta}\left|\mathbf{S}_{f}\right|
$$

where $\theta_{f i}$ is $\theta_{i}$ linearly interpolated from cell centres to faces, $\nabla_{S} \pi=\nabla \pi \cdot \mathbf{S}_{f}, \pi_{c}$ and $\pi_{N}$ are the values of Exner pressure in the cells either side of face $f$ and $\delta$ is the distance between the cell centres. The interpolation of $\theta$ in this term means that the discretisation uses Lorenz staggering in the vertical.

\section{A.3. Transfer Terms in the Momentum Equation}

The transfer terms on the right hand side of the momentum equation (16) are calculated at cell centres and so need to be linearly interpolated onto faces, and their dot products with $\mathbf{S}_{f}$ are taken in order to calculate the rate of change of $U_{i}$. This is not done using conservative interpolation so momentum could be created or destroyed when mass is transferred.

\section{A.4. Advection of $\sigma_{i} \rho_{i}$}

Here we consider the discretisation of the advection term of the continuity equation (11). The mass transfer terms that appear in the $\theta$ and momentum equations $(16,15)$ involve division by $\sigma_{i} \rho_{i}$ so for stability, $\sigma_{i} \rho_{i}$ should remain positive. Therefore $\sigma_{i} \rho_{i}$ should be advected using a monotonic scheme. However for energy conservation and for consistency between the continuity and pressure equation (which uses the continuity equation, see section 3.2.5), $\rho_{i}$ should be advected using centred differencing. The advection term of eqn 11 is therefore discretised using Gauss's divergence theorem as:

$$
\nabla \cdot\left(\sigma_{i} \rho_{i} \mathbf{u}_{i}\right) \approx \frac{1}{V} \sum_{f \in c} \sigma_{i a} \rho_{i f} U_{f}
$$

where $\rho_{i f}=\lambda \rho_{i c}+(1-\lambda) \rho_{i N}$ using the same interpolation weights and notation as for eqn (56) and where $\sigma_{i a}$ is interpolated from cell centre values of $\sigma_{i}$ to faces using the monotonic OpenFOAM van-Leer advection scheme:

$$
\sigma_{i a}=\sigma_{i u}+\phi\left(\sigma_{i f}-\sigma_{i u}\right)
$$

where $\sigma_{i u}$ is the value of $\sigma_{i}$ in the upwind cell, $\sigma_{i f}$ is the linearly interpolated value and $\phi$ is the van-Leer limiter function:

$$
\phi=\frac{r+|r|}{1+|r|}, \quad r=2 \frac{\left(\mathbf{x}_{d}-\mathbf{x}_{u}\right) \cdot \nabla_{u} \sigma_{i}}{\sigma_{i d}-\sigma_{i u}}-1
$$

where $\mathbf{x}_{u}$ and $\mathbf{x}_{d}$ are the locations of the upwind and downwind cell centres, $\sigma_{i d}$ is the value of $\sigma_{i}$ in the downwind cell and $\nabla_{u} \sigma_{i}$ is the gradient of $\sigma_{i}$ calculated in the upwind cell using Gauss's divergence theorem. This discretisation is only monotonic when $\rho_{i}$ is sufficiently smooth in the direction of flow which is usually achieved in low Mach number flows.

\section{A.5. Advection of $\theta_{i}$}

Here we consider the advection term in the temperature equation (15). For consistency with advection in the momentum equation (eqn 59), $\theta$ advection is calculated in advective form using finite volume operators (using Gauss's theorem):

$$
\mathbf{u}_{i} \cdot \nabla \theta_{i} \approx \frac{1}{V}\left(\sum_{f \in c} \theta_{a i} U_{i}-\theta_{i} \sum_{f \in c} U_{i}\right)
$$

where $\theta_{a}$ is interpolated from cell centres to faces using the OpenFOAM van-Leer advection scheme as for $\sigma_{i}$. 